\title{
Aluminum Chloride-Catalyzed C-Alkylation of Pyrrole and Indole with Chalcone and Bis-Chalcone Derivatives
}

\author{
Meliha Burcu Gürdere**a, Oguz Özbek ${ }^{a}$, Mustafa Ceylan ${ }^{a}$ \\ ${ }^{a}$ Department of Chemistry, Faculty of Arts and Sciences, Gaziosmanpasa \\ University, 60250, Tokat, Turkey
}

\section{SUPPLEMENTAL MATERIAL}

\section{General procedure for the synthesis of 3a-i:}

A mixture of pyrrole (2) $(10 \mathrm{mmol})$ and chalcone (1a-i) $(1 \mathrm{mmol})$ and $\mathrm{AlCl}_{3}(10 \%$, mol) was stirred at room temperature for $24 \mathrm{~h}$. until starting material disappeared on TLC. After completion of the reaction, the catalyst was separated by filtration. The reaction mixture was then diluted with water and extracted with $\mathrm{CHCl}_{3}$. Subsequently, the organic layer was dried over anhydrous sodium sulfate. Evaporating of the solvent under reduced pressure gave the corresponding viscous oil products.

1,3-di(furan-2-yl)-3-(1H-pyrrol-2-yl)propan-1-one (3a). Viscous oil; Yield (84\%). ${ }^{1} \mathrm{H}$ NMR (400 MHz, $\left.\mathrm{CDCl}_{3}\right) \delta(\mathrm{ppm}): 8.63(\mathrm{~s}, 1 \mathrm{H}), 7.59$ (br s, 1H), 7.40 (br s, 1H), 7.23 (d, $J=$ $3.2 \mathrm{~Hz}, 1 \mathrm{H}), 6.71(\mathrm{t}, J=1.2 \mathrm{~Hz}, 1 \mathrm{H}), 6.56(\mathrm{t}, J=1.6 \mathrm{~Hz}, 1 \mathrm{H}), 6.33$ (t, $J=1.4 \mathrm{~Hz}, 1 \mathrm{H}), 6.18-$ $6.14(\mathrm{~m}, 2 \mathrm{H}), 5.98$ (br s, 1H), 4.89 (t, $J=7.0, \mathrm{~Hz}, 1 \mathrm{H}), 3.58(\mathrm{dd}, J=6.4 ; 4.0 ; 2.4 \mathrm{~Hz}, 2 \mathrm{H})$. ${ }^{13} \mathrm{C}-\mathrm{NMR}\left(100 \mathrm{MHz}, \mathrm{CDCl}_{3}\right) \delta(\mathrm{ppm}): 187.4,157.4,152.5,151.5,146.7,141.6,131.5,117.2$, 112.5, 110.3 (2C), 105.5, 105.3, 44.1, 33.4. Anal. Cald for $\mathrm{C}_{15} \mathrm{H}_{13} \mathrm{NO}_{3}$ : C, 70.58; $\mathrm{H}, 5.13 ; \mathrm{N}$, 5.49. Found: C, 70.46; H, 5.09; N, 5.37.

1-(furan-2-yl)-3-phenyl-3-(1H-pyrrol-2-yl)propan-1-one (3b). Viscous oil; Yield (89\%). ${ }^{1} \mathrm{H}-\mathrm{NMR}\left(400 \mathrm{MHz}, \mathrm{CDCl}_{3}\right) \delta(\mathrm{ppm}): 8.36(\mathrm{~s}, 1 \mathrm{H}), 7.33(\mathrm{~s}, 4 \mathrm{H}), 7.28(\mathrm{~m}, 2 \mathrm{H}), 7.21(\mathrm{~d}, J=$ $3.6 \mathrm{~Hz}, 1 \mathrm{H}), 6.67$ (br s, 1H), 6.54 (br s, $1 \mathrm{H}), 6.13$ ( br s, $1 \mathrm{H}), 5.92$ ( br s, $1 \mathrm{H}), 4.78$ (t, $J=$ 
6.8, Hz, 1H), 3.69 (dd, $J=16.8,8.4 \mathrm{~Hz}, 1 \mathrm{H}), 3.48(\mathrm{dd}, J=17.0,6,0 \mathrm{~Hz}, 1 \mathrm{H}) .{ }^{13} \mathrm{C}-\mathrm{NMR}(100$ $\left.\mathrm{MHz}, \mathrm{CDCl}_{3}\right) \delta(\mathrm{ppm}): 190.4,156.8,148.5,140.6,130.3,128.1$ (2C), 126.9 (2C), 125.8, 121.0, 118.4, 112.6, 108.2, 107.6, 42.5, 38.9. Anal. Cald for $\mathrm{C}_{17} \mathrm{H}_{15} \mathrm{NO}_{2}$ : C, 79.96, H, 5.70; N, 5.28. Found: C, 78.98, H, 5.67; N, 5.16.

1-(furan-2-yl)-3-(4-methoxyphenyl)-3-(1H-pyrrol-2-yl)propan-1-one (3c). Viscous oil; Yield (95\%). ${ }^{1} \mathrm{H}-\mathrm{NMR}\left(400 \mathrm{MHz}, \mathrm{CDCl}_{3}\right) \delta(\mathrm{ppm}): 8.33$ (br s, $\left.1 \mathrm{H}\right), 7.57$ (d, $\left.J=7.6 \mathrm{~Hz}, 1 \mathrm{H}\right)$, $7.23(\mathrm{~d}, J=8.4 \mathrm{~Hz}, 2 \mathrm{H}), 7.19(\mathrm{~d}, J=3.6 \mathrm{~Hz}, 1 \mathrm{H}), 6.87(\mathrm{~d}, J=8.8 \mathrm{~Hz}, 2 \mathrm{H}), 6.70(\mathrm{br} \mathrm{s}, 1 \mathrm{H})$, $6.53(\mathrm{~d}, J=1.6 \mathrm{~Hz}, 1 \mathrm{H}), 6.13(\mathrm{dd}, J=5.6,2.8 \mathrm{~Hz}, 1 \mathrm{H}), 5.92($ br s, $1 \mathrm{H}), 4.72(\mathrm{t}, J=7.2 \mathrm{~Hz}$, 1H), $3.82(\mathrm{~s}, 3 \mathrm{H}), 3.65(\mathrm{dd}, J=16.8,8.0 \mathrm{~Hz}, 1 \mathrm{H}), 3.46(\mathrm{dd}, J=16.8,6.4 \mathrm{~Hz}, 1 \mathrm{H}) .{ }^{13} \mathrm{C}-\mathrm{NMR}$ $\left(100 \mathrm{MHz}, \mathrm{CDCl}_{3}\right) \delta(\mathrm{ppm}): 198.5,158.1,152.3,146.5,135.4,129.8,129.0(2 \mathrm{C}), 117.4$ 117.1, 114.0 (2C), 112.3, 107.8, 105.3, 55.2, 44.9, 38.3. Anal. Cald for $\mathrm{C}_{18} \mathrm{H}_{17} \mathrm{NO}_{3}$ : C, 73.20; H, 5.80; N, 4.74. Found: C, 73.98; H, 5.67; N, 4.69.

1-(furan-2-yl)-3-(1H-pyrrol-2-yl)-3-p-tolylpropan-1-one (3d). Viscous oil; Yield (93\%). 1H-NMR (400 MHz, $\left.\mathrm{CDCl}_{3}\right) \delta(\mathrm{ppm}): 8.34(\mathrm{~s}, 1 \mathrm{H}), 7.59(\mathrm{~d}, J=0.8 \mathrm{~Hz}, 1 \mathrm{H}), 7.22(\mathrm{~d}, J=8.0$ $\mathrm{Hz}, 2 \mathrm{H}), 7.16(\mathrm{~d}, J=7.6 \mathrm{~Hz}, 2 \mathrm{H}), 7.11(\mathrm{~d}, J=3.6 \mathrm{~Hz}, 1 \mathrm{H}), 6.67(\mathrm{~d}, J=1.6 \mathrm{~Hz}, 1 \mathrm{H}), 6.54$ (dd, $J=3.6,1.6 \mathrm{~Hz}, 1 \mathrm{H}), 6.13(\mathrm{dd}, J=6.0,2.8 \mathrm{~Hz}, 1 \mathrm{H}), 5.93($ br s, $1 \mathrm{H}), 4.75(\mathrm{t}, J=6.8 \mathrm{~Hz}$, 1H), $3.68(\mathrm{dd}, J=17.0,8.0 \mathrm{~Hz}, 1 \mathrm{H}), 3.47(\mathrm{dd}, J=16.8,6.0 \mathrm{~Hz}, 1 \mathrm{H}), 2.36(\mathrm{~s}, 3 \mathrm{H}) .{ }^{13} \mathrm{C}-\mathrm{NMR}$ $\left(100 \mathrm{MHz}, \mathrm{CDCl}_{3}\right) \delta$ (ppm): 192.6, 152.8, 148.8, 137.6, 135.3, 130.1, 129.1 (2C), 127.3 (2C), 121.6, 118.1, 112.5, 108.5, 108.2, 42.4, 38.9, 24.6. Anal. Cald for $\mathrm{C}_{17} \mathrm{H}_{18} \mathrm{NO}_{2}$ : C, 77.40; $\mathrm{H}$, 6.13; N, 5.01. Found: C, 76.98; H, 6.07; N, 4.98.

3-(1H-pyrrol-2-yl)-1,3-di(thiophen-2-yl)propan-1-one (3e). Viscous oil; Yield (91\%). ${ }^{1} \mathrm{H}$ NMR (400 MHz, $\left.\mathrm{CDCl}_{3}\right) \delta(\mathrm{ppm}): 8.48$ (br s, $\left.1 \mathrm{H}\right), 7.80(\mathrm{~d}, J=2.8 \mathrm{~Hz}, 1 \mathrm{H}), 7.70(\mathrm{~d}, J=4.4$ Hz,1H), $7.24(\mathrm{~d}, J=4.4 \mathrm{~Hz}, 1 \mathrm{H}), 7.18(\mathrm{~d}, J=4.0 \mathrm{~Hz}, 1 \mathrm{H}), 7.00 \quad(\mathrm{~m}, 2 \mathrm{H}), 6.73($ br s, $1 \mathrm{H})$, 6.21 (br s, 1H), 6.10 (br s, 1H), 5.09 (t, $J=7.2 \mathrm{~Hz}, 1 \mathrm{H}), 3.79$ (dd, $J=16.8,7.2 \mathrm{~Hz}, 1 \mathrm{H}), 3.69$ (dd, $J=16.8,6.0 \mathrm{~Hz}, 1 \mathrm{H}) .{ }^{13} \mathrm{C}-\mathrm{NMR}\left(100 \mathrm{MHz}, \mathrm{CDCl}_{3}\right) \delta(\mathrm{ppm}): 191.2,146.6,144.0,140.1$, 
$136.6,134.2,133.1,132.5,131.9,128.9,120.8,120.4,119.8,46.8,35.1$. Anal. Cald for $\mathrm{C}_{15} \mathrm{H}_{13} \mathrm{NOS}_{2}: \mathrm{C}, 62.69 ; \mathrm{H}, 4.56 ; \mathrm{N}, 4.87$. Found: C, 62.58; H, 4.47; N, 4.79.

1-(4-chlorophenyl)-3-(1H-pyrrol-2-yl)-3-(thiophen-2-yl)propan-1-one (3f).

Viscous oil; Yield (89\%). ${ }^{1} \mathrm{H}-\mathrm{NMR}\left(400 \mathrm{MHz}^{\mathrm{CDCl}}{ }_{3}\right) \delta(\mathrm{ppm}): 8.38$ (br s, 1H), 7.56 (br s, 1H), 7.28-7.16 (m, 7H), 6.52 (br s, 1H), 5.71 ( br s, $1 \mathrm{H}), 4.63$ (t, $J=7.2 \mathrm{~Hz}, 1 \mathrm{H}), 3.55$ (dd, $J$ $=17.0,7.6 \mathrm{~Hz}, 1 \mathrm{H}), 3.30(\mathrm{dd}, J=16.8,6.0 \mathrm{~Hz}, 1 \mathrm{H}) .{ }^{13} \mathrm{C}-\mathrm{NMR}\left(100 \mathrm{MHz}, \mathrm{CDCl}_{3}\right) \delta(\mathrm{ppm})$ : $192.1,142.8,138.6,134.5,133.8,131.4,130.3,129.2(2 \mathrm{C}), 128.5(2 \mathrm{C}), 128.1,117.9,108.5$, 107.6, 43.9, 39.8. Anal. Cald for $\mathrm{C}_{17} \mathrm{H}_{14} \mathrm{ClNOS}$ : C, 64.65; H, 4.47; N, 4.44. Found: C, 64.58; $\mathrm{H}, 4.37 ; \mathrm{N}, 4.41$.

3-(1H-pyrrol-2-yl)-3-(thiophen-2-yl)-1-p-tolylpropan-1-one (3g). Viscous oil; Yield (93\%). ${ }^{1} \mathrm{H}-\mathrm{NMR}\left(400 \mathrm{MHz}, \mathrm{CDCl}_{3}\right) \delta(\mathrm{ppm}): 8.41$ (br s, $\left.1 \mathrm{H}\right), 7.83(\mathrm{~d}, J=8.8 \mathrm{~Hz}, 2 \mathrm{H}), 7.63(\mathrm{~d}, J=$ $8.4 \mathrm{~Hz}, 2 \mathrm{H}), 7.20(\mathrm{~d}, J=5.2 \mathrm{~Hz}, 1 \mathrm{H}) .6 .92(\mathrm{~m}, 2 \mathrm{H}), 6.69$ (br s, 1H), 6.29 (br s, 1H), 6.08 (br $\mathrm{s}, 1 \mathrm{H}), 5.11(\mathrm{t}, J=6.4 \mathrm{~Hz}, 1 \mathrm{H}), 3.78(\mathrm{dd}, J=17.6,7.6 \mathrm{~Hz}, 1 \mathrm{H}), 3.66(\mathrm{dd}, J=17.4,6.0 \mathrm{~Hz}$, 1H), 2.39 (s, 3H). ${ }^{13} \mathrm{C}-\mathrm{NMR}\left(100 \mathrm{MHz}, \mathrm{CDCl}_{3}\right) \delta(\mathrm{ppm}): 199.9,144.8,142.6,133.5,130.8$, 129.0 (2C), 128.8 (2C), 127.1, 126.9, 118.8, 108.4, 108.1, 44.1, 37.5, 24.0. Anal. Cald for $\mathrm{C}_{18} \mathrm{H}_{17} \mathrm{NOS}$ : C, 73.19; H, 5.80; N, 4.74. Found: C, 73.08; H, 5.78; N, 4.67.

3-(4-methoxyphenyl)-3-(1H-pyrrol-2-yl)-1-(thiophen-2-yl)propan-1-one (3h). Viscous oil;

Yield (84\%). ${ }^{1} \mathrm{H}-\mathrm{NMR}\left(400 \mathrm{MHz}, \mathrm{CDCl}_{3}\right) \delta(\mathrm{ppm}): 8.52$ (br s, $\left.1 \mathrm{H}\right), 7.98(\mathrm{~d}, J=8.0 \mathrm{~Hz}, 2 \mathrm{H})$, $7.11(\mathrm{~m}, 1 \mathrm{H}), 7.00(\mathrm{~d}, J=8.0 \mathrm{~Hz}, 2 \mathrm{H}), 6.89(\mathrm{~m}, 1 \mathrm{H}), 6.70(\mathrm{~m}, 1 \mathrm{H}), 6.24(\mathrm{~m}, 1 \mathrm{H}), 6.18$ $(\mathrm{m}, 1 \mathrm{H}), 6.04(\mathrm{~m}, 1 \mathrm{H}), 5.15(\mathrm{t}, J=6.8 \mathrm{~Hz}, 1 \mathrm{H}), 3.91(\mathrm{~s}, 3 \mathrm{H}), 3.81(\mathrm{dd}, J=17.2,8.0 \mathrm{~Hz}, 2 \mathrm{H}$, A), $3.69(\mathrm{dd}, J=17.2,6.0 \mathrm{~Hz}, 2 \mathrm{H}, \mathrm{B}) .{ }^{13} \mathrm{C}-\mathrm{NMR}\left(100 \mathrm{MHz}, \mathrm{CDCl}_{3}\right) \delta(\mathrm{ppm}): 197.0,163.7$, $146.7,134.1,130.4(2 \mathrm{C}), 128.5,126.7,124.7,123.9,117.1,113.8(2 \mathrm{C}), 108.2,107.0,55.5$, 46.0, 34.8. Anal. Cald for $\mathrm{C}_{18} \mathrm{H}_{17} \mathrm{NO}_{2} \mathrm{~S}$ : C, 69,43; H, 5,50; N, 4,50. Found: C, 64.38; H, 5.47; $\mathrm{N}, 44$ 
3-(1H-pyrrol-2-yl)-1-(thiophen-2-yl)-3-p-tolylpropan-1-one (3i). Viscous oil; Yield (86\%).

${ }^{1} \mathrm{H}-\mathrm{NMR}\left(400 \mathrm{MHz}, \mathrm{CDCl}_{3}\right) \delta(\mathrm{ppm}): 8.38(\mathrm{br} \mathrm{s}, 1 \mathrm{H}), 7.76(\mathrm{~d}, J=3.2 \mathrm{~Hz}, 1 \mathrm{H}), 7.65(\mathrm{~d}, J=$ $4.8 \mathrm{~Hz}, 1 \mathrm{H}), 7.24-7.15(\mathrm{~m}, 5 \mathrm{H}), 6.69$ (br s, $1 \mathrm{H}), 6.23$ (d, $J=2.8 \mathrm{~Hz}, 1 \mathrm{H}), 5.97$ (br s, $1 \mathrm{H})$, $4.79(\mathrm{t}, J=6.8 \mathrm{~Hz}, 1 \mathrm{H}), 3.78(\mathrm{dd}, J=16.8,8.0 \mathrm{~Hz}, 1 \mathrm{H}), 3.57(\mathrm{dd}, J=16.8,6.0 \mathrm{~Hz}, 1 \mathrm{H}), 2.39$ (s, 3H). ${ }^{13} \mathrm{C}-\mathrm{NMR}\left(100 \mathrm{MHz}, \mathrm{CDCl}_{3}\right) \delta(\mathrm{ppm}): 191.9,144.1,139.6,136.5,134.5,134.0$, $132.1,129.3,129.2,128.1,127.9,117.1,108.3,107.8,105.4,45.9,39.1,21.0$. Anal. Cald for $\mathrm{C}_{18} \mathrm{H}_{17} \mathrm{NOS}$ : C, 73.19; H, 5.80; N, 4.74. Found: C, 75.98; H, 5.77; N, 4.68.

\section{General procedure for the synthesis of $4 a-f$}

To a mixture of pyrrole (2) $(1 \mathrm{mmol})$ and chalcone $(\mathbf{1})(3 \mathrm{mmol})$ in $\mathrm{CH}_{3} \mathrm{CN}(5 \mathrm{~mL})$ and $\mathrm{AlCl}_{3}$ $(10 \%, \mathrm{w} / \mathrm{w})$ was added and the mixture was stirred at room temperature for $3 \mathrm{~h}$. The reaction was followed by TLC. After completion of the reaction, the catalyst was separated by filtration. The reaction mixture was then diluted with water and extracted with $\mathrm{CHCl}_{3}$. Subsequently, the organic layer was dried over anhydrous sodium sulfate. Evaporating of the solvent under reduced pressure gave the corresponding viscous oil products.

3,3'-(1H-pyrrole-2,5-diyl)bis(1,3-bis(4-methoxyphenyl)propan-1-one) (4a).Viscous oil; Yield (85\%). ${ }^{1} \mathrm{H}-\mathrm{NMR}\left(400 \mathrm{MHz}, \mathrm{CDCl}_{3}\right) \delta$ (ppm): 8.53 (br s, $\left.1 \mathrm{H}\right), 8.05$ (d, J=8.8 Hz, 4H), $7.87(\mathrm{~d}, J=8.4 \mathrm{~Hz}, 4 \mathrm{H}), 6.95(\mathrm{~d}, J=8.8 \mathrm{~Hz}, 4 \mathrm{H}), 6.84(\mathrm{~d}, J=8.4 \mathrm{~Hz}, 4 \mathrm{H}), 5.79(\mathrm{~d}, J=2.4$ $\mathrm{Hz}, 1 \mathrm{H}), 5.75(\mathrm{~d}, J=4.0 \mathrm{~Hz}, 1 \mathrm{H}), 4.76(\mathrm{t}, J=6.8 \mathrm{~Hz}, 2 \mathrm{H}), 3.79(\mathrm{~s}, 12 \mathrm{H}), 3.65$ (dd, $J=17.2$, $7.6 \mathrm{~Hz}, 2 \mathrm{H}), 3.45(\mathrm{dd}, J=16.8,6.4 \mathrm{~Hz}, 2 \mathrm{H}) .{ }^{13} \mathrm{C}-\mathrm{NMR}\left(100 \mathrm{MHz}, \mathrm{CDCl}_{3}\right) \delta(\mathrm{ppm}): 197.4$ (2C), 163.5 (2C), 158.1 (2C), 138.1 (2C), 135.5 (2C), 130.7 (2C), 128.9 (4C), 127.7 (4C), 114.4 (4C), 114.0 (4C), 107.7 (2C), 55.3 (2C), 55.0 (2C), 44.8 (2C), 38.9 (2C). Anal. Cald for $\mathrm{C}_{38} \mathrm{H}_{37} \mathrm{NO}_{6}:$ C, 75.60; H, 6.18; N, 2.32. Found: C, 74.98; H, 6.07; N, 2.28.

3,3'-(1H-pyrrole-2,5-diyl)bis(3-(4-chlorophenyl)-1-phenylpropan-1-one) (4b). Viscous oil; Yield (93\%). ${ }^{1} \mathrm{H}-\mathrm{NMR}\left(400 \mathrm{MHz}, \mathrm{CDCl}_{3}\right) \delta$ (ppm): 8.59 (br s, $\left.1 \mathrm{H}\right), 8.04$ (d, J=8.0 Hz, 4H), $7.90(\mathrm{~d}, J=7.2 \mathrm{~Hz}, 2 \mathrm{H}), 7.62(\mathrm{~d}, J=7.2 \mathrm{~Hz}, 2 \mathrm{H}), 7.52(\mathrm{~d}, J=8.0 \mathrm{~Hz}, 2 \mathrm{H}), 7.43(\mathrm{~d}, J=8.0$ 
$\mathrm{Hz}, 2 \mathrm{H}), 7.38$ (d, $J=8.0 \mathrm{~Hz}, 2 \mathrm{H}), 7.21(\mathrm{~d}, J=7.2 \mathrm{~Hz}, 4 \mathrm{H}), 5.74(\mathrm{~d}, J=5.6 \mathrm{~Hz}, 2 \mathrm{H}), 4.79$ (t, $J$ $=6.8 \mathrm{~Hz}, 2 \mathrm{H}), 3.79(\mathrm{dd}, J=17.6,7.2 \mathrm{~Hz}, 2 \mathrm{H}), 3.58(\mathrm{dd}, J=16.8,6.4 \mathrm{~Hz}, 2 \mathrm{H}) .{ }^{13} \mathrm{C}-\mathrm{NMR}$ $\left(100 \mathrm{MHz}, \mathrm{CDCl}_{3}\right) \delta(\mathrm{ppm}): 198.5$ (2C), 143.3 (2C), 141.8 (2C), 137.9 (2C), 136.4 (2C), 133.8 (2C), 129.7 (4C), 128.7 (4C), 128.5 (4C), 128.1 (4C), 108.0 (2C), 45.0 (2C), 38.8 (2C). Anal. Cald for $\mathrm{C}_{34} \mathrm{H}_{27} \mathrm{Cl}_{2} \mathrm{NO}_{2}$ : C, 73.91; H, 4.93; N, 2.54. Found: C, 73.77; H, 4.81; N, 2.48 .

3,3'-(1H-pyrrole-2,5-diyl)bis(1,3-di(furan-2-yl)propan-1-one) (4c). Viscous oil; Yield (76\%). ${ }^{1} \mathrm{H}-\mathrm{NMR}\left(400 \mathrm{MHz}, \mathrm{CDCl}_{3}\right) \delta$ (ppm): 8.65 (br s, 1H), 7.57 (br s, 2H), 7.33 (br s, 2H), $7.19(\mathrm{~d}, J=3.2 \mathrm{~Hz}, 2 \mathrm{H}), 6.53-6.51(\mathrm{~m}, 2 \mathrm{H}), 6.28($ br s, $2 \mathrm{H}), 6.08(\mathrm{t}, J=3.2 \mathrm{~Hz}, 2 \mathrm{H})$, $5.79(\mathrm{t}, J=3.2 \mathrm{~Hz}, 2 \mathrm{H}), 4.79(\mathrm{t}, J=7.2 \mathrm{~Hz}, 2 \mathrm{H}), 3.71(\mathrm{~d}, J=7.2 \mathrm{~Hz}, 4 \mathrm{H}) .{ }^{13} \mathrm{C}-\mathrm{NMR}(100$ $\left.\mathrm{MHz}, \mathrm{CDCl}_{3}\right) \delta(\mathrm{ppm}): 187.1(2 \mathrm{C}), 155.2(2 \mathrm{C}), 152.5,146.5$ (2C), 141.5 (2C), $141.3,130.9$ (2C), 117.5, $112.3(2 \mathrm{C}), 112.1,110.2$ (2C), $106.0(2 \mathrm{C}), 105.2$ (2C), 44.0, 42.9, 33.4, 31.8. Anal. Cald for $\mathrm{C}_{26} \mathrm{H}_{21} \mathrm{NO}_{6}$ : C, 70.42; H, 4.77; N, 3.16. Found: C, 70.38; H, 4.62; N, 3.12.

3,3'-(1H-pyrrole-2,5-diyl)bis(1,3-di(thiophen-2-yl)propan-1-one) (4d). Viscous oil; Yield (82\%). ${ }^{1} \mathrm{H}-\mathrm{NMR}\left(400 \mathrm{MHz}, \mathrm{CDCl}_{3}\right) \delta$ (ppm): 8.53 (br s, $\left.1 \mathrm{H}\right), 7.74(\mathrm{~d}, J=6.8 \mathrm{~Hz}, 4 \mathrm{H}), 7.72$ $(\mathrm{d}, J=6.8 \mathrm{~Hz}, 4 \mathrm{H}), 7.48(\mathrm{~d}, J=5.2 \mathrm{~Hz}, 2 \mathrm{H}), 7.42-7.39(\mathrm{~m}, 2 \mathrm{H}), 5.98(\mathrm{br} \mathrm{s}, 2 \mathrm{H}), 5.08(\mathrm{t}, J=$ $7.2 \mathrm{~Hz}, 2 \mathrm{H}), 3.68(\mathrm{dd}, J=17.2,5.2 \mathrm{~Hz}, 2 \mathrm{H}), 3.61(\mathrm{dd}, J=17.0,6.4 \mathrm{~Hz}, 2 \mathrm{H}) .{ }^{13} \mathrm{C}-\mathrm{NMR}(100$ $\left.\mathrm{MHz}, \mathrm{CDCl}_{3}\right) \delta(\mathrm{ppm}): 190.5(2 \mathrm{C}), 145.6(2 \mathrm{C}), 144.0$ (2C), $135.6(2 \mathrm{C}), 134.2(2 \mathrm{C}), 133.0$ (2C), 130.9 (2C), 128.6 (2C), 120.8 (2C), 120.4 (2C), 110.3 (2C), 46.8 (2C), 35.1 (2C). Anal. Cald for $\mathrm{C}_{26} \mathrm{H}_{21} \mathrm{NO}_{2} \mathrm{~S}_{4}$ : C, 61.51; H, 4.17; N, 2.76. Found: C, 61.36; H, 4.02; N, 2.73.

3,3'-(1H-pyrrole-2,5-diyl)bis(3-(4-chlorophenyl)-1-(thiophen-2-yl)propan-1-one)

(4e).

Viscous oil; Yield (85\%). ${ }^{1} \mathrm{H}-\mathrm{NMR}\left(400 \mathrm{MHz}, \mathrm{CDCl}_{3}\right) \delta$ (ppm): 8.47 (br s, 1H), 7.55 (br s, 2H), 7.28-7.14 (m, 10H), 6.51-6.48 (m, 2H), 5.75-5.72 (m, 2H), $4.74(\mathrm{t}, J=6.8 \mathrm{~Hz}, 2 \mathrm{H})$, $3.55(\mathrm{dd}, J=16.8,7.6 \mathrm{~Hz}, 2 \mathrm{H}), 3.35(\mathrm{dd}, J=11.6,6.0 \mathrm{~Hz}, 2 \mathrm{H}) .{ }^{13} \mathrm{C}-\mathrm{NMR}\left(100 \mathrm{MHz}, \mathrm{CDCl}_{3}\right)$ $\delta(\mathrm{ppm}): 187.5(2 \mathrm{C}), 152.5,146.6(2 \mathrm{C}), 146.2,141.3,133.4(2 \mathrm{C}), 132.4,129.3$ (4C), 128.9 
(4C), 117.6 (2C), 112.4 (2C), 105.4 (2C), 105.2 (2C), 44.5, 43.5, 38.7, 38.6. Anal. Cald for $\mathrm{C}_{30} \mathrm{H}_{23} \mathrm{Cl}_{2} \mathrm{NO}_{2} \mathrm{~S}_{2}: \mathrm{C}, 63.83 ; \mathrm{H}, 4.11 ; \mathrm{N}, 2.48$. Found: C, 63.78; H, 4.05; N, 2.42 .

3,3'-(1H-pyrrole-2,5-diyl)bis(1-(4-chlorophenyl)-3-(thiophen-2-yl)propan-1-one)

(4f).

Viscous oil; Yield (84\%). ${ }^{1} \mathrm{H}-\mathrm{NMR}\left(400 \mathrm{MHz}, \mathrm{CDCl}_{3}\right) \delta(\mathrm{ppm}): 8.44$ (br s, $\left.1 \mathrm{H}\right), \quad 7.86-7.84$ $(\mathrm{m}, 4 \mathrm{H}), \quad 7.45-7.38(\mathrm{~m}, 4 \mathrm{H}), \quad 7.17-7.15(\mathrm{~m}, 2 \mathrm{H}), \quad 6.95-6.90(\mathrm{~m}, 4 \mathrm{H}), 5.85(\mathrm{dd}, J=12.0,6.8$ $\mathrm{Hz}, 2 \mathrm{H}), 5.03(\mathrm{t}, J=6.8 \mathrm{~Hz}, 2 \mathrm{H}), 3.70(\mathrm{dd}, J=17.2,5.2 \mathrm{~Hz}, 2 \mathrm{H}), 3.58(\mathrm{dd}, J=17.2,6.0 \mathrm{~Hz}$, 2H). ${ }^{13} \mathrm{C}-\mathrm{NMR}\left(100 \mathrm{MHz}, \mathrm{CDCl}_{3}\right) \delta$ (ppm): 189.2 (2C), $146.8(2 \mathrm{C}), 136.2(2 \mathrm{C}), 132.4(2 \mathrm{C})$, 130.3 (4C), 128.9 (4C), 127.6 (2C), 123.4 (2C), 121.7 (2C), 107.4 (2C), 105.2 (2C), 44.8, 43.5, 37.6, 37.1. Anal. Cald for $\mathrm{C}_{30} \mathrm{H}_{23} \mathrm{Cl}_{2} \mathrm{NO}_{2} \mathrm{~S}_{2}$ : C, 63.83; H, 4.11; N, 2.48. Found: C, 63.66; H, 3.99; N, 2.38.

\section{General Procedure for the Synthesis of Bis-Chalcone Derivatives (5a-g)}

To a solution of acetophenone derivatives $(2 \mathrm{mmol})$ in EtOH $(20 \mathrm{ml})$ was added $\mathrm{NaOH}(2.5$ M, $6 \mathrm{ml})$. The mixture was stirred for $3 \mathrm{~min}$ and was added teraftaldehit $(1 \mathrm{mmol})$ and was stirred at r.t. for $4 \mathrm{~h}$. After the reaction, the precipitate was filtered and washed with ethanol several times. The solid was crystallized by methylene chloride / hexane (3/7).

3,3'-(1,4-phenylene)bis(1-(thiophene-3-yl)prop-2-en-1-one (5f). Yield (90\%); mp: 219-221 ${ }^{\circ} \mathrm{C} .{ }^{1} \mathrm{H}-\mathrm{NMR}\left(400 \mathrm{MHz}, \mathrm{CDCl}_{3}\right) \delta(\mathrm{ppm}): 8.85(\mathrm{~s}, 2 \mathrm{H}), 7.97(\mathrm{~s}, 4 \mathrm{H}), 7.95-7.91(\mathrm{~d}, J=15.6 \mathrm{~Hz}$, $2 \mathrm{H}), 7.76-7.72(\mathrm{~d}, J=15.6 \mathrm{~Hz}, 2 \mathrm{H}), 7.70-7.69(\mathrm{~m}, 4 \mathrm{H}) .{ }^{13} \mathrm{C}-\mathrm{NMR}\left(100 \mathrm{MHz}, \mathrm{CDCl}_{3}\right) \delta$ (ppm): 183.3, 143.3, 142.5, 137.0, 135.0, 129.7, 128.2, 127.5, 124.5. IR Spektrumu $\left(\mathrm{KCl}, \mathrm{cm}^{-}\right.$ $\left.{ }^{1}\right)$ : $3077,1656,1600,1511,1423,1396,1230,1203,1072,1035,981,804,713,690$. Anal. calc. for $\mathrm{C}_{20} \mathrm{H}_{14} \mathrm{O}_{2} \mathrm{~S}_{2}: \mathrm{C}, 68.54 ; \mathrm{H}, 4.03$. Found: C, 68.28; H, 4.02.

\section{General procedure for the synthesis of 6a-g and 8a-g}

To a solution of pyrrole (2) $(8 \mathrm{mmol})$ or indole (7) $(8 \mathrm{mmol})$ and bis-chalcone (5a-g) (1 mmol) in $\mathrm{CH}_{3} \mathrm{CN}(5 \mathrm{~mL})$ was added $\mathrm{AlCl}_{3}(10 \%$, w/w $)$ and the mixture was stirred at room temperature for $24 \mathrm{~h}$. The reaction was followed by TLC. After completion of the reaction, 
the catalyst was separated by filtration. The reaction mixture was then diluted with water and extracted with $\mathrm{CHCl}_{3}$. Subsequently, the organic layer was dried over anhydrous sodium sulfate. Evaporating of the solvent under reduced pressure gave the corresponding solid products. The crude products were purified by recrystallization from $n$-hexane- $\mathrm{CHCl}_{3}$.

3,3'-(1,4-phenylene)bis(1-phenyl-3-(1H-pyrrol-2-yl)propan-1-one) (6a). Yield (85\%); mp: 212-214 ${ }^{\circ} \mathrm{C} .{ }^{1} \mathrm{H}-\mathrm{NMR}\left(400 \mathrm{MHz}, \mathrm{CDCl}_{3}\right.$ ) $\delta$ (ppm): 8.43 (br s, 2H), 7.97 (d, $J=7.2 \mathrm{~Hz}, 4 \mathrm{H}$ ), 7.60-7.57 (m, 2H), $7.48(\mathrm{t}, J=7.2 \mathrm{~Hz}, 4 \mathrm{H}), 7.27(\mathrm{~s}, 4 \mathrm{H}), 6.68(\mathrm{~s}, 2 \mathrm{H}), 6.11(\mathrm{~s}, 2 \mathrm{H}), 5.87(\mathrm{~s}$, 2H), $4.77(\mathrm{~d}, J=7.2 \mathrm{~Hz}, 2 \mathrm{H}), 3.83-3.79(\mathrm{dd}, J=8.0,17.6 \mathrm{~Hz}, 2 \mathrm{H}), 3.64-3.58(\mathrm{dd}, J=5.6$, $17.6 \mathrm{~Hz}, 2 \mathrm{H}) .{ }^{13} \mathrm{C}-\mathrm{NMR}\left(100 \mathrm{MHz}, \mathrm{CDCl}_{3}\right) \delta(\mathrm{ppm}): 197.7$ (2C), 141.4 (2C), 139.8 (2C), $135.1(2 \mathrm{C}), \quad 134.1$ (2C), 129.5 (4C), 128.9 (4C), 128.3 (4C), 117.2 (2C), 107.9 (2C), 105.4 (2C), 45.1 (2C), 39.0 (2C). Anal. Cald for $\mathrm{C}_{32} \mathrm{H}_{28} \mathrm{~N}_{2} \mathrm{O}_{2}$ : C, 81.33; H, 5.97; N, 5.93. Found: C, 81.27; H, 5.91; N, 5.86.

\section{3,3'-(1,4-phenylene)bis(1-(4-chlorophenyl)-3-(1H-pyrrol-2-yl)propan-1-one) (6b).}

Yield (89\%); mp: $232-233{ }^{\circ} \mathrm{C} .{ }^{1} \mathrm{H}-\mathrm{NMR}\left(400 \mathrm{MHz}, \mathrm{CDCl}_{3}\right) \delta(\mathrm{ppm}): 8.82(\mathrm{~s}, 2 \mathrm{H}), 7.80-7.78$ $(\mathrm{dd}, J=2.0,6.8 \mathrm{~Hz}, 4 \mathrm{H}), 7.35-7.33(\mathrm{dd}, J=2.0,6.8 \mathrm{~Hz}, 4 \mathrm{H}), 7.13(\mathrm{~s}, 4 \mathrm{H}), 6.56-6.55(\mathrm{dd}, J=$ 1.6, $2.4 \mathrm{~Hz}, 2 \mathrm{H}), 5.98-5.96(\mathrm{dd}, J=2.4,5.8 \mathrm{~Hz}, 2 \mathrm{H}), 5.77-5.76(\mathrm{dd}, J=1.2,2.0 \mathrm{~Hz}, 2 \mathrm{H})$, $4.65(\mathrm{t}, J=6.8 \mathrm{~Hz}, 2 \mathrm{H}), 3.68-3.62(\mathrm{dd}, J=7.6,17.2 \mathrm{~Hz}, 2 \mathrm{H}), 3.49-3.44(\mathrm{dd}, J=6.4,17.2$ $\mathrm{Hz}, 2 \mathrm{H}) .{ }^{13} \mathrm{C}-\mathrm{NMR}\left(100 \mathrm{MHz}, \mathrm{CDCl}_{3}\right) \delta$ (ppm): 198.1 (2C), 141.6 (2C), 139.9 (2C), 135.2 (2C), 134.2 (2C), 129.1 (4C), 128.8 (4C), 128.4 (4C), 128.2 (2C), 117.5 (2C), 107.8 (2C), 45.1 (2C), 39.2 (2C). Anal. Cald for $\mathrm{C}_{32} \mathrm{H}_{26} \mathrm{Cl}_{2} \mathrm{~N}_{2} \mathrm{O}_{2}$ : C, 70.98; H, 4.84; N, 5.17. Found: C, 70.77; H, 4.81; N, 5.08.

\section{3,3'-(1,4-phenylene)bis(1-(4-methoxyphenyl)-3-(1H-pyrrol-2-yl)propan-1-one) (6c).}

Yield (74\%); mp: 205-207 ${ }^{\circ} \mathrm{C} .{ }^{1} \mathrm{H}-\mathrm{NMR}\left(400 \mathrm{MHz}, \mathrm{CDCl}_{3}\right) \delta$ (ppm): 8.39 (br s, 2H), 7.94 (d, $J=8.8 \mathrm{~Hz}, 4 \mathrm{H}), 7.26(\mathrm{~s}, 4 \mathrm{H}), 6.93(\mathrm{~d}, J=9.2 \mathrm{~Hz}, 4 \mathrm{H}), 6.67-6.66(\mathrm{dd}, J=2.4,4 \mathrm{~Hz}, 2 \mathrm{H})$ 6.09-6.07 (dd, $J=2.4,5.8 \mathrm{~Hz}, 2 \mathrm{H}), 5.81(\mathrm{~d}, J=8.4 \mathrm{~Hz}, 2 \mathrm{H}), 4.77-4.73(\mathrm{dd}, J=5.6,7.8 \mathrm{~Hz}$, 
2H), $3.86(\mathrm{~s}, 6 \mathrm{H}), 3.77-3.75(\mathrm{dd}, J=8.4,17.2 \mathrm{~Hz}, 2 \mathrm{H}), 3.57-3.51(\mathrm{dd}, J=5.6,17.4 \mathrm{~Hz}$, 2H). ${ }^{13} \mathrm{C}-\mathrm{NMR}\left(100 \mathrm{MHz}, \mathrm{CDCl}_{3}\right) \delta(\mathrm{ppm}): 197.6(2 \mathrm{C}), 163.6(2 \mathrm{C}), 141.5(2 \mathrm{C}), 134.7(2 \mathrm{C})$, 130.4 (4C), 129.9 (2C), 128.3 (4C), 117.0 (2C), 113.7 (4C), 107.7 (2C), 105.4 (2C), 55.4 (2C), 44.9 (2C), 39.1 (2C). Anal. Cald for $\mathrm{C}_{34} \mathrm{H}_{32} \mathrm{~N}_{2} \mathrm{O}_{4}$ : C, 76.67; H, 6.06; N, 5.26. Found: C, 76.58; H, 5.99; N, 5.16 .

3,3'-(1,4-phenylene)bis(3-(1H-pyrrol-2-yl)-1-(p-tolyl)propan-1-one) (6d). Yield (71\%); mp: $208-210{ }^{\circ} \mathrm{C} .{ }^{1} \mathrm{H}-\mathrm{NMR}\left(400 \mathrm{MHz}, \mathrm{CDCl}_{3}\right) \delta(\mathrm{ppm}): 8.34$ (br s, $\left.2 \mathrm{H}\right), 7.87$ (d, $J=8.0 \mathrm{~Hz}$, 4H), $7.27(\mathrm{~s}, 4 \mathrm{H}), 6.86(\mathrm{~d}, J=8.6 \mathrm{~Hz}, 4 \mathrm{H}), 6.67-6.66(\mathrm{~m}, 2 \mathrm{H}), 6.11-6.10(\mathrm{~m}, 2 \mathrm{H}), 5.84(\mathrm{~m}$, 2H), $4.75(\mathrm{t}, J=5.2 \mathrm{~Hz}, 2 \mathrm{H}), 3.77-3.75(\mathrm{dd}, J=7.6,16.8 \mathrm{~Hz}, 2 \mathrm{H}), 3.60-3.54(\mathrm{dd}, J=5.6$, $17.4 \mathrm{~Hz}, 2 \mathrm{H}), 2.43(\mathrm{~s}, 6 \mathrm{H}) .{ }^{13} \mathrm{C}-\mathrm{NMR}\left(100 \mathrm{MHz}, \mathrm{CDCl}_{3}\right) \delta(\mathrm{ppm}): 197.6(2 \mathrm{C}), 163.6(2 \mathrm{C})$, $141.5(2 \mathrm{C}), 134.7(2 \mathrm{C}), \quad 130.0(4 \mathrm{C}), 128.8(2 \mathrm{C}), 128.5$ (4C), $117.0(2 \mathrm{C}), 113.7$ (4C), 107.7 (2C), 105.4 (2C), 55.4 (2C), 44.9 (2C), 39.1 (2C). Anal. Cald for $\mathrm{C}_{34} \mathrm{H}_{32} \mathrm{~N}_{2} \mathrm{O}_{2}$ : C, 81.57; H, 6.44; N, 5.60. Found: C, 81.48; H, 6.36; N, 5.53.

3,3'-(1,4-phenylene)bis(3-(1H-pyrrol-2-yl)-1-(thiophen-2-yl)propan-1-one) (6e). Yield (89\%); mp: $206-208{ }^{\circ} \mathrm{C} .{ }^{1} \mathrm{H}-\mathrm{NMR}\left(400 \mathrm{MHz}, \mathrm{CDCl}_{3}\right) \delta$ (ppm): 10.59 (s, 2H), 8.08 (d, $J=3.6$ $\mathrm{Hz}, 2 \mathrm{H}), 7.97(\mathrm{~d}, J=8.6 \mathrm{~Hz}, 2 \mathrm{H}), 7.23(\mathrm{t}, J=4.8 \mathrm{~Hz}, 2 \mathrm{H}), 7.18(\mathrm{~s}, 4 \mathrm{H}), 6.53$ (t, $J=7.6 \mathrm{~Hz}$, $2 \mathrm{H}), 5.84(\mathrm{~s}, 4 \mathrm{H}), 4.56(\mathrm{t}, J=7.6 \mathrm{~Hz}, 2 \mathrm{H}), 3.79-3.72(\mathrm{dd}, J=16.8,4.4 \mathrm{~Hz}, 2 \mathrm{H}), 3.55-3.42$ (dd, $J=16.8,6.8 \mathrm{~Hz}, 2 \mathrm{H}) .{ }^{13} \mathrm{C}-\mathrm{NMR}\left(100 \mathrm{MHz}, \mathrm{CDCl}_{3}\right) \delta(\mathrm{ppm}): 191.5$ (2C), 144.5 (2C), $142.4(2 \mathrm{C}), 135.3(2 \mathrm{C}), 134.3(2 \mathrm{C}), 133.9(2 \mathrm{C}), 129.2(2 \mathrm{C}), 127.7(2 \mathrm{C}), 117.8(2 \mathrm{C}), 117.0$ (2C), 107.5 (2C), 107.4 (2C), $104.6(2 \mathrm{C}), 44.4$ (2C). Anal. Cald for $\mathrm{C}_{28} \mathrm{H}_{24} \mathrm{~N}_{2} \mathrm{O}_{2} \mathrm{~S}_{2}$ : C, 69.39; H, 4.99; N, 5.78. Found: C, 69.18; H, 4.91; N, 5.66.

3,3'-(1,4-phenylene)bis(3-(1H-pyrrol-2-yl)-1-(thiophen-3-yl)propan-1-one) (6f). Yield (76\%); mp: $202-204{ }^{\circ} \mathrm{C} .{ }^{1} \mathrm{H}-\mathrm{NMR}\left(400 \mathrm{MHz}, \mathrm{CDCl}_{3}\right) \delta(\mathrm{ppm}): 8.33$ (s, 2H), 7.85 (d, $J=8.0$ $\mathrm{Hz}, 2 \mathrm{H}), 7.23(\mathrm{~s}, 4 \mathrm{H}), 7.10(\mathrm{~m}, 2 \mathrm{H}), 7.03(\mathrm{~m}, 2 \mathrm{H}), 6.70(\mathrm{~m}, 2 \mathrm{H}), 6.15(\mathrm{~m}, 2 \mathrm{H}) .5,83(\mathrm{~s}, 2 \mathrm{H})$, $4.76(\mathrm{t}, J=5.6 \mathrm{~Hz}, 2 \mathrm{H}), 3.80-3.74(\mathrm{dd}, J=17.2,7.2 \mathrm{~Hz}, 2 \mathrm{H}), 3.59-3.54(\mathrm{dd}, J=17.6,5.6 \mathrm{~Hz}$, 
2H). ${ }^{13} \mathrm{C}-\mathrm{NMR}\left(100 \mathrm{MHz}, \mathrm{CDCl}_{3}\right) \delta(\mathrm{ppm}): 198.7$ (2C), 144.1 (2C), $134.4(2 \mathrm{C}), 129.3$ (4C), 128.8 (2C), 128.2 (4C), 127.3 (2C), 117.0 (2C), 108.2 (2C), 107.8 (2C), 45.1 (2C), 39.0 (2C). Anal. Cald for $\mathrm{C}_{28} \mathrm{H}_{24} \mathrm{~N}_{2} \mathrm{O}_{2} \mathrm{~S}_{2}$ : C, 69.39; H, 4.99; N, 5.78. Found: C, 69.28; H, 4.89; N, 5.71 . 3,3'-(1,4-phenylene)bis(1-(naphthalen-2-yl)-3-(1H-pyrrol-2-yl)propan-1-one) (6g). Yield (81\%); mp: $236-238^{\circ} \mathrm{C} .{ }^{1} \mathrm{H}-\mathrm{NMR}$ (400 MHz, $\left.\mathrm{CDCl}_{3}\right) \delta$ (ppm): 8.15 (br s, 2H), 7.98 (dd, $J=$ 8.2, 4.8 Hz, 2H), 7.92-7.87 (m, 2H), $7.77(\mathrm{t}, J=6.4 \mathrm{~Hz}, 2 \mathrm{H}), 7.54-7.51(\mathrm{~m}, 4 \mathrm{H}), 7.47-7.42(\mathrm{~m}$, 4H), 7.23 (s, 4H), 6.67-6.64 (m, 2H), 6.14-6.12 (m, 2H), 5.94-5.93 (m, 2H), 4.81 (t, $J=7.6$ $\mathrm{Hz}, 2 \mathrm{H}), 3.84$ (ddd, $J=7.6,6.4 \mathrm{~Hz}, 2 \mathrm{H}), 3.66$ (ddd, $J=6.6,3.2 \mathrm{~Hz}, 2 \mathrm{H}) .{ }^{13} \mathrm{C}-\mathrm{NMR}(100$ $\left.\mathrm{MHz}, \mathrm{CDCl}_{3}\right) \delta(\mathrm{ppm}): 198.5$ (2C), 143.3 (2C), 141.8 (2C), 137.9 (2C), 136.4 (2C), 133.8 (2C), 129.7 (4C), 128.7 (6C), 128.5 (4C), 128.1 (4C), 118.0 (2C), 108.1 (2C), 107.7 (2C), 45.0 (2C), 38.8 (2C). Anal. Cald for $\mathrm{C}_{40} \mathrm{H}_{32} \mathrm{~N}_{2} \mathrm{O}_{2}$ : C, 83.89; H, 5.63; N, 4.89. Found: C, 83.78; $\mathrm{H}, 5.59 ; \mathrm{N}, 4.79$.

3,3'-(1,4-phenylene)bis(3-(1H-indol-3-yl)-1-phenylpropan-1-one) (8a). Yield (75\%); mp: 230-232 ${ }^{\circ} \mathrm{C} .{ }^{1} \mathrm{H}-\mathrm{NMR}\left(400 \mathrm{MHz}, \mathrm{CDCl}_{3}\right) \delta(\mathrm{ppm}): 10.81$ (s, 2H), 7.97-7.94 (m, 4H), 7.60$7.58(\mathrm{~m}, 2 \mathrm{H}), 7.46(\mathrm{t}, J=7.6 \mathrm{~Hz}, 4 \mathrm{H}), 7.39-7.36(\mathrm{~m}, 2 \mathrm{H}), 7.26(\mathrm{~s}, 8 \mathrm{H}), 7.01-6.97(\mathrm{~m}, 2 \mathrm{H})$, 6.86-6.82 (m, 2H), 4.79 (t, $J=7.2 \mathrm{~Hz}, 2 \mathrm{H}), 3.93-3.87(\mathrm{dd}, J=7.6,17.2 \mathrm{~Hz}, 2 \mathrm{H}), 3.69-3.63$ (dd, $J=6.8,17.2 \mathrm{~Hz}, 2 \mathrm{H}) .{ }^{13} \mathrm{C}-\mathrm{NMR}\left(100 \mathrm{MHz}, \mathrm{CDCl}_{3}\right) \delta(\mathrm{ppm}): 199.3$ (2C), $143.6(2 \mathrm{C})$, 137.8 (2C), 137.2 (2C), 134.0 (2C), 129.5 (4C), 128.9 (4C), 128.4 (4C), 127.3 (2C), 122.7 (2C), 121.8 (2C), 119.6 (2C), 119.1 (2C), 118.9 (2C), 112.2 (2C), 45.3 (2C), 38.1 (2C). Anal. Cald for $\mathrm{C}_{40} \mathrm{H}_{32} \mathrm{~N}_{2} \mathrm{O}_{2}:$ C, 83.89, H, 5.63; N, 4.89. Found: C, 83.77; H, 5.58; N, 4.81.

3,3'-(1,4-phenylene)bis(1-(4-chlorophenyl)-3-(1H-indol-3-yl)propan-1-one) (8b). Viscous oil; Yield (69\%). ${ }^{1} \mathrm{H}-\mathrm{NMR}\left(400 \mathrm{MHz}, \mathrm{CDCl}_{3}\right) \delta(\mathrm{ppm}): 8.27$ (br s, 2H), 7.69 (d, J=8.0 Hz, 2H), $7.43(\mathrm{~d}, J=8.0 \mathrm{~Hz}, 2 \mathrm{H}), 7.24-7.20(\mathrm{~m}, 8 \mathrm{H}), 7.18(\mathrm{~s}, 4 \mathrm{H}), 7.16-7.10(\mathrm{~m}, 2 \mathrm{H}), 6.82-6.78$ (t, $J=7.2 \mathrm{~Hz}, 2 \mathrm{H}), 6.72-6.70(\mathrm{~d}, J=7.6 \mathrm{~Hz}, 2 \mathrm{H}), 5.29(\mathrm{t}, J=8.8 \mathrm{~Hz}, 2 \mathrm{H}), 3.55-3.48(\mathrm{dd}, J=$ 15.6, $8.8 \mathrm{~Hz}, 2 \mathrm{H}), 3.28-3.22$ ( dd, $J=15.6,8.4 \mathrm{~Hz}, 2 \mathrm{H}) .{ }^{13} \mathrm{C}-\mathrm{NMR}\left(100 \mathrm{MHz}, \mathrm{CDCl}_{3}\right)$ 
$\begin{array}{lllllll}\delta(\mathrm{ppm}): & 188.7 & (2 \mathrm{C}), & 135.7 & (2 \mathrm{C}), & 127.8 & (2 \mathrm{C}),\end{array}$ (4C), 122.0 (4C), $121.2(2 \mathrm{C}), 120.7$ (4C), $119.6(4 \mathrm{C}), 111.3(4 \mathrm{C}), 109.3(2 \mathrm{C}), 102.6(4 \mathrm{C}), 112$ $.2(2 \mathrm{C}), 56.4(2 \mathrm{C}), 37.6(2 \mathrm{C})$. Anal. Cald for $\mathrm{C}_{40} \mathrm{H}_{30} \mathrm{Cl}_{2} \mathrm{~N}_{2} \mathrm{O}_{2}: \mathrm{C}, 74.88, \mathrm{H}, 4.71 ; \mathrm{N}, 4.37$. Found: C, 74.67; H, 4.48; N, 4.21.

3,3'-(1,4-phenylene)bis(1-(4-methoxyphenyl)-3-(1H-indol-3-yl)propan-1-one) (8c). Yield (73\%); mp: 228-230 ${ }^{\circ} \mathrm{C} .{ }^{1} \mathrm{H}-\mathrm{NMR}\left(400 \mathrm{MHz}, \mathrm{CDCl}_{3}\right) \delta(\mathrm{ppm}): 8.04$ (s, 2H), 7.98-7.96 (d, J= $8.8 \mathrm{~Hz}, 2 \mathrm{H}), 7.41-7.39(\mathrm{~d}, J=8.0 \mathrm{~Hz}, 2 \mathrm{H}), 7.26(\mathrm{~s}, 4 \mathrm{H}), 7.23-7.10(\mathrm{~m}, 8 \mathrm{H}), 6.97-6.95(\mathrm{~d}, J=$ $8.8 \mathrm{~Hz}, 2 \mathrm{H}), 6.80-6.76(\mathrm{t}, J=7.2 \mathrm{~Hz}, 2 \mathrm{H}), 6.71-6.69(\mathrm{~d}, J=7.6 \mathrm{~Hz}, 2 \mathrm{H}), 5.28(\mathrm{t}, J=8.8 \mathrm{~Hz}$, 2H), $3.93(\mathrm{~s}, 6 \mathrm{H}), 3.53-3.47(\mathrm{dd}, J=15.6,9.2 \mathrm{~Hz}, 2 \mathrm{H}), 3.27-3.21(\mathrm{dd}, J=15.4,8.4 \mathrm{~Hz}, 2 \mathrm{H})$. ${ }^{13} \mathrm{C}-\mathrm{NMR}\left(100 \mathrm{MHz}, \mathrm{CDCl}_{3}\right) \delta(\mathrm{ppm}): 188.2$ (2C), 163.7 (2C), 136.8 (2C), $131.0(2 \mathrm{C}), 128.9$ (2C), 127.5 (2C), 122.8 (4C), 122.3 (2C), 121.8 (2C), 121.3 (4C), 119.6 (4C), 118.8 (2C), 114.0 (2C), $111.4(2 \mathrm{C}), 109.3$ (2C), 56.4 (2C), 55.6 (2C), 37.7 (2C). Anal. Cald for $\mathrm{C}_{42} \mathrm{H}_{36} \mathrm{~N}_{2} \mathrm{O}_{4}: \mathrm{C}, 79.72, \mathrm{H}, 5.73 ; \mathrm{N}, 4.43$. Found: $\mathrm{C}, 79.67 ; \mathrm{H}, 5.66 ; \mathrm{N}, 4.31$.

3,3'-(1,4-phenylene)bis(1-(4-tolyphenyl)-3-(1H-indol-3-yl)propan-1-one) (8d). Yield (81\%); mp: 232-235 ${ }^{\circ} \mathrm{C} . \quad{ }^{1} \mathrm{H}-\mathrm{NMR} \quad\left(400 \mathrm{MHz}, \mathrm{CDCl}_{3}\right) \quad \delta \quad(\mathrm{ppm}): 8.03 \quad(\mathrm{~s}, \quad 2 \mathrm{H})$, 7.64-7.61 (m, 2H), $7.38(\mathrm{~d}, J=8.0 \mathrm{~Hz}, 2 \mathrm{H}), 7.25(\mathrm{~s}, 4 \mathrm{H}), 7.23-7.10(\mathrm{~m}, 10 \mathrm{H}), 6.80-6.76(\mathrm{t}, J=$ $7.2 \mathrm{~Hz}, 2 \mathrm{H}), 6.72-6.70(\mathrm{~d}, J=8.0 \mathrm{~Hz}, 2 \mathrm{H}), 5.29(\mathrm{t}, J=8.4 \mathrm{~Hz}, 2 \mathrm{H}), 3.54-3.47(\mathrm{dd}, J=15.6$, $9.2 \mathrm{~Hz}, 2 \mathrm{H}), 3.27-3.21(\mathrm{dd}, J=15.6,8.4 \mathrm{~Hz}, 2 \mathrm{H}), 2.43(\mathrm{~s}, 6 \mathrm{H}) .{ }^{13} \mathrm{C}-\mathrm{NMR}\left(100 \mathrm{MHz}, \mathrm{CDCl}_{3}\right)$

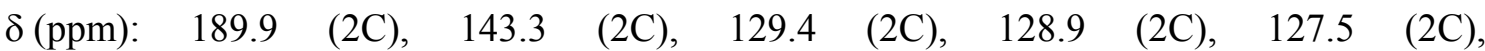
124.2 (2C), 123.0 (2C), 122.3 (4C), 121.2 (4C), $119.6(4 \mathrm{C}), 119.3$ (2C), $119.1(2 \mathrm{C}), 111.4(2$ C), $109.2(2 \mathrm{C}), 56.4(2 \mathrm{C}), 37.6(2 \mathrm{C}), 21,7$ (2C). Anal. Cald for $\mathrm{C}_{42} \mathrm{H}_{36} \mathrm{~N}_{2} \mathrm{O}_{2}: \mathrm{C}, 83.97 ; \mathrm{H}$, 6.04; N, 4.66. Found: C, 83.77; H, 5.98; N, 4.51.

3,3'-(1,4-phenylene)bis(1-(furan-2-yl)-3-(1H-indol-3-yl)propan-1-one) (8e). Yield (77\%); mp: $278-281{ }^{\circ} \mathrm{C} .{ }^{1} \mathrm{H}-\mathrm{NMR}\left(400 \mathrm{MHz}, \mathrm{CDCl}_{3}\right) \delta(\mathrm{ppm}): 10.35(\mathrm{~s}, 2 \mathrm{H}), \delta=7.76(\mathrm{~s}, 2 \mathrm{H}), 7.56$ 
(t, $J=0.8 \mathrm{~Hz}, 2 \mathrm{H}), 7.23(\mathrm{t}, J=7.2 \mathrm{~Hz}, 4 \mathrm{H}), 7.14-7.12(\mathrm{~m}, 4 \mathrm{H}), 6.98-6.93(\mathrm{~m}, 4 \mathrm{H}), 6.80(\mathrm{t}, J=$ $7.2 \mathrm{~Hz}, 2 \mathrm{H}), 6.44-6.43(\mathrm{~m}, 2 \mathrm{H}), 4.80-4.77$ (t, $J=8.0 \mathrm{~Hz} 2 \mathrm{H}), 3.61-3.55$ (dd, $J=7.6,15.2 \mathrm{~Hz}$ 2H), 3.39-3.32 (ddd, $J=3.6,7.6,16.0 \mathrm{~Hz}, 2 \mathrm{H}) .{ }^{13} \mathrm{C}-\mathrm{NMR}\left(100 \mathrm{MHz}, \mathrm{CDCl}_{3}\right) \delta(\mathrm{ppm}): 187.2$ $\begin{array}{llllll}(2 \mathrm{C}), & 152.6 & (2 \mathrm{C}), & 146.7 & (2 \mathrm{C}), & 142.3\end{array}$ $136.7(2 \mathrm{C}), 127.7(4 \mathrm{C}), 126.5(2 \mathrm{C}), 121.8(2 \mathrm{C}), 121.3(2 \mathrm{C}), 119.0(2 \mathrm{C}), 118.6(2 \mathrm{C}), 117.7(2$ C), $112.4(2 \mathrm{C}), 111.5(2 \mathrm{C}), 44.8(2 \mathrm{C}), 37.8(2 \mathrm{C}), 37.7(2 \mathrm{C})$. Anal. Cald for $\mathrm{C}_{36} \mathrm{H}_{28} \mathrm{~N}_{2} \mathrm{O}_{4}$ : C, 78.24; H, 5.11; N, 5.07. Found: C, 78.17; H, 5.08; N, 4.98.

\section{3,3'-(1,4-phenylene)bis(3-(1H-indol-3-yl)-1-(thiophen-2-yl)propan-1-one) (8f).}

Yield (83\%); mp: 208-211 ${ }^{\circ} \mathrm{C} .{ }^{1} \mathrm{H}-\mathrm{NMR}\left(400 \mathrm{MHz}, \mathrm{CDCl}_{3}\right) \delta$ (ppm): 8.19 (br s, 2H), 7.71 (d, $J=5.2 \mathrm{~Hz}, 2 \mathrm{H}), 7.63(\mathrm{~d}, J=7.6 \mathrm{~Hz}, 2 \mathrm{H}), 7.45(\mathrm{~d}, J=5.2 \mathrm{~Hz}, 2 \mathrm{H}), 7.39(\mathrm{~d}, J=8.0 \mathrm{~Hz}, 2 \mathrm{H})$, 7.26-7.00 (m, 6H), $6.81(\mathrm{t}, J=7.2 \mathrm{~Hz}, 2 \mathrm{H}), 6.71(\mathrm{~d}, J=7.6 \mathrm{~Hz}, 2 \mathrm{H}), 6.61-6.59(\mathrm{~m}, 2 \mathrm{H}), 5.29$ $(\mathrm{t}, J=8.8 \mathrm{~Hz}, 2 \mathrm{H}), 3.55-3.48(\mathrm{dd}, J=9.2,15.6 \mathrm{~Hz}, 2 \mathrm{H}), 3.28-3.22(\mathrm{dd}, J=8.4,15.6 \mathrm{~Hz}, 2 \mathrm{H})$.

${ }^{13} \mathrm{C}-\mathrm{NMR}\left(100 \mathrm{MHz}, \quad \quad \mathrm{CDCl}_{3}\right) \delta(\mathrm{ppm}): \quad 198.2 \quad \quad(2 \mathrm{C}), \quad 127.4$ (2C), 125.7 (2C), 124.7 (2C), 124.1 (2C), 122.3 (2C), 121.9 (4C), 121.2 (2C), 120.7 (4C), 119 $.6(4 \mathrm{C}), 119.5(2 \mathrm{C}), 119.4(2 \mathrm{C}), 118.8(2 \mathrm{C}), 56.4(2 \mathrm{C}), 37.6(2 \mathrm{C})$. Anal. $\quad$ Cald $\quad$ for $\mathrm{C}_{36} \mathrm{H}_{28} \mathrm{~N}_{2} \mathrm{O}_{2} \mathrm{~S}_{2:}$ C, 73.94; H, 4.83; N, 4.79. Found: C, 73.87; H, 4.7 8; N, 4.72.

3,3'-(1,4-phenylene)bis(3-(1H-indol-3-yl)-1-(naphthalen-2-yl)propan-1-one) (8g). Yield (78\%); mp: 225-228 ${ }^{\circ} \mathrm{C} .{ }^{1} \mathrm{H}-\mathrm{NMR}\left(400 \mathrm{MHz}, \mathrm{CDCl}_{3}\right) \delta$ (ppm): 10.33 (s, 2H), 7.97 (t, J= 8.8 $\mathrm{Hz}, 2 \mathrm{H}), 7.89-7.86(\mathrm{dd}, J=3.6,8.2 \mathrm{~Hz}, 2 \mathrm{H}), 7.77(\mathrm{~d}, J=8.4 \mathrm{~Hz}, 2 \mathrm{H}), 7.70-7.67(\mathrm{~m}, 2 \mathrm{H}), 7.39-$ $7.33(\mathrm{~m}, 4 \mathrm{H}), 7.27-7.20(\mathrm{~m}, 6 \mathrm{H}), 7.13(\mathrm{~s}, 4 \mathrm{H}), 6.99-6.95(\mathrm{~m}, 4 \mathrm{H}), 6.83-6.80(\mathrm{dd}, J=5.2,8.0$ $\mathrm{Hz}, 2 \mathrm{H}), 4.87-4.84(\mathrm{t}, J=8.0 \mathrm{~Hz}, 2 \mathrm{H}), 3.79-3.73(\mathrm{dd}, J=1.2,9.2 \mathrm{~Hz}, 2 \mathrm{H}), 3.68-3.65$ (dd, $J=$ 5.2, $8.0 \mathrm{~Hz}, 2 \mathrm{H}) .{ }^{13} \mathrm{C}-\mathrm{NMR}(100 \mathrm{MHz}, \mathrm{CDCl} 3) \delta(\mathrm{ppm}): 203.0$ (2C), 142.4 (2C), 136.8 (2C), 136.6 (2C), 133.7 (2C), 132.1 (2C), 129.7 (2C), 128.3 (2C), 127.9 (4C), 127.4 (2 C), 127.0 (2C), 126.6 (2C), 126.4 (2C), 125.5 (2C), 124.6 (2C), 121.8 (2C), 121.3 (2C), 119.0 
(2C), 118.6 (2C), 117.8 (2C), 11.5 (2C), 49.4 (2C), 39.4 (2C). Anal. Cald for $\mathrm{C}_{48} \mathrm{H}_{36} \mathrm{~N}_{2} \mathrm{O}_{2:}$ C, 85.69; H, 5.39; N, 4.16. Found: C, 85.57; H, 5.32; N, 4.13. 
Figure S1. ${ }^{1} \mathrm{H}$ NMR and ${ }^{13} \mathrm{C}$ NMR spectra of compound $\mathbf{5 f}$ Figure S2. ${ }^{1} \mathrm{H}$ NMR and ${ }^{13} \mathrm{C}$ NMR spectra of compound $\mathbf{6 b}$ Figure S3. ${ }^{1} \mathrm{H}$ NMR and ${ }^{13} \mathrm{C}$ NMR spectra of compound $\mathbf{6 c}$ Figure S4. ${ }^{1} \mathrm{H}$ NMR and ${ }^{13} \mathrm{C}$ NMR spectra of compound $6 \mathbf{e}$ Figure S5. ${ }^{1} \mathrm{H}$ NMR and ${ }^{13} \mathrm{C}$ NMR spectra of compound $\mathbf{8 a}$ Figure S1. ${ }^{1} \mathrm{H}$ NMR and ${ }^{13} \mathrm{C}$ NMR spectra of compound 8e 


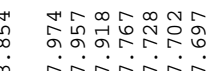

- rinitín
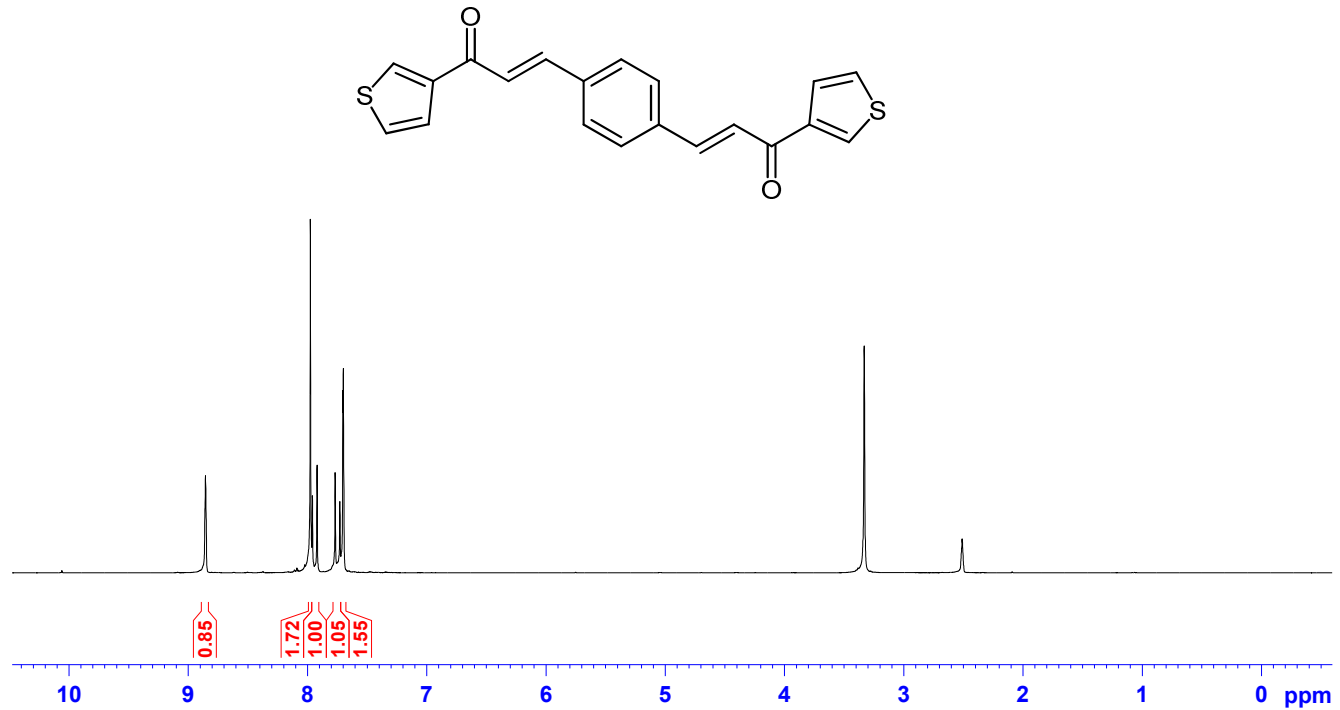

Figure S1. ${ }^{1} \mathrm{H}$ NMR spectrum (400 MHz, DMSO) of $\mathbf{5 f}$

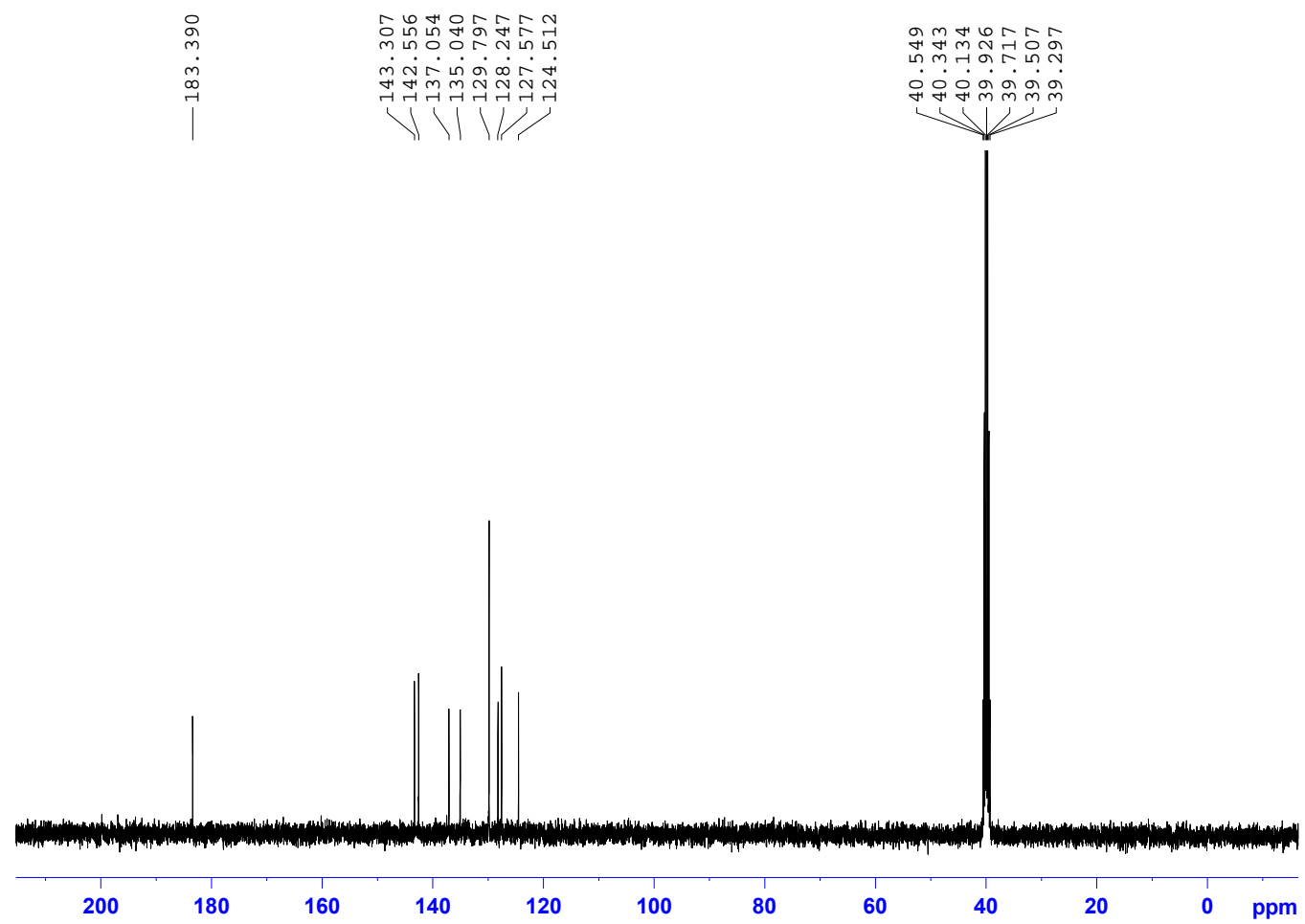

Figure S1. ${ }^{13} \mathrm{C}$ NMR spectrum $(100 \mathrm{MHz}, \mathrm{DMSO})$ of $\mathbf{5 f}$ 

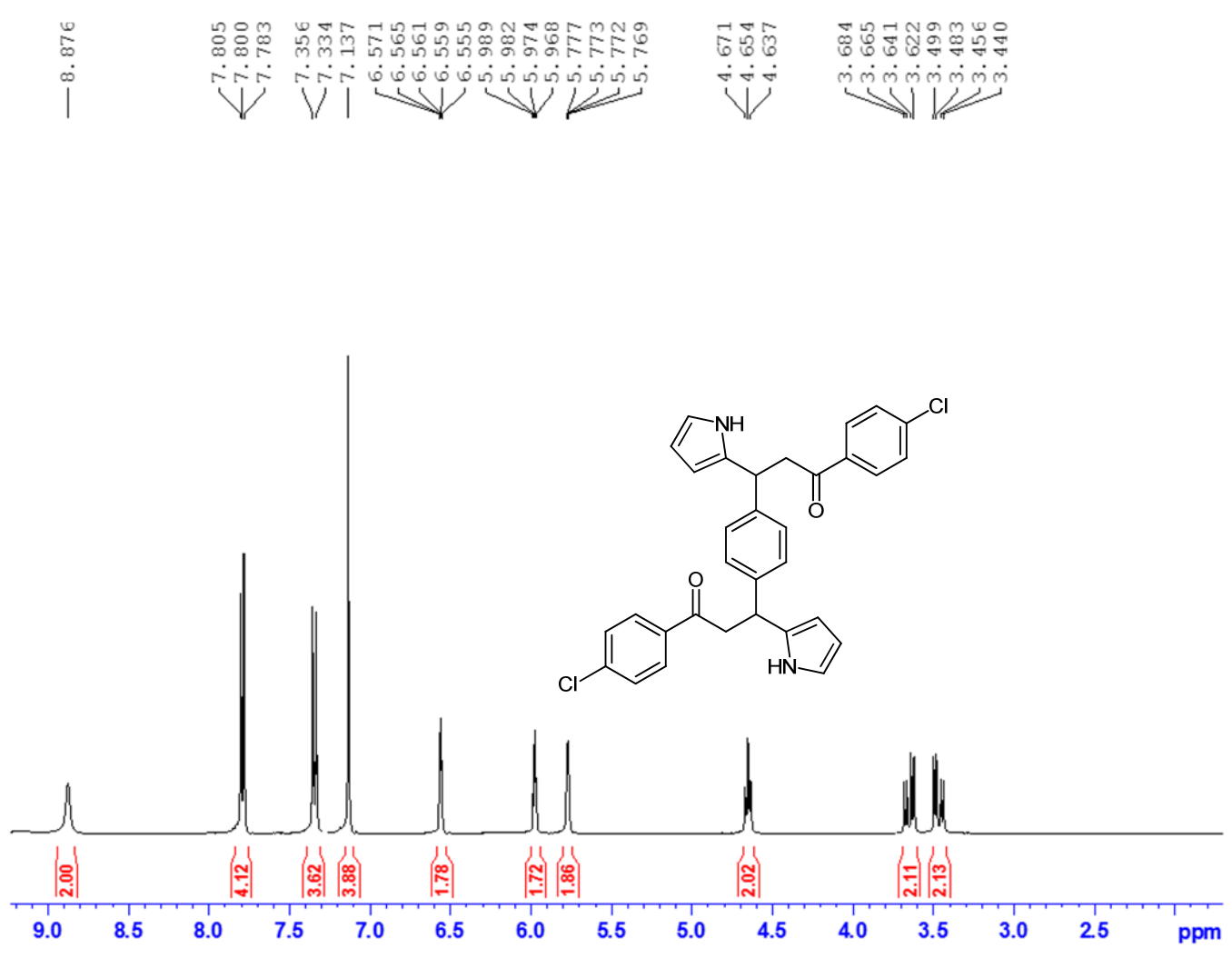

Figure S2. ${ }^{1} \mathrm{H}$ NMR spectrum (400 MHz, DMSO) of $\mathbf{6 b}$

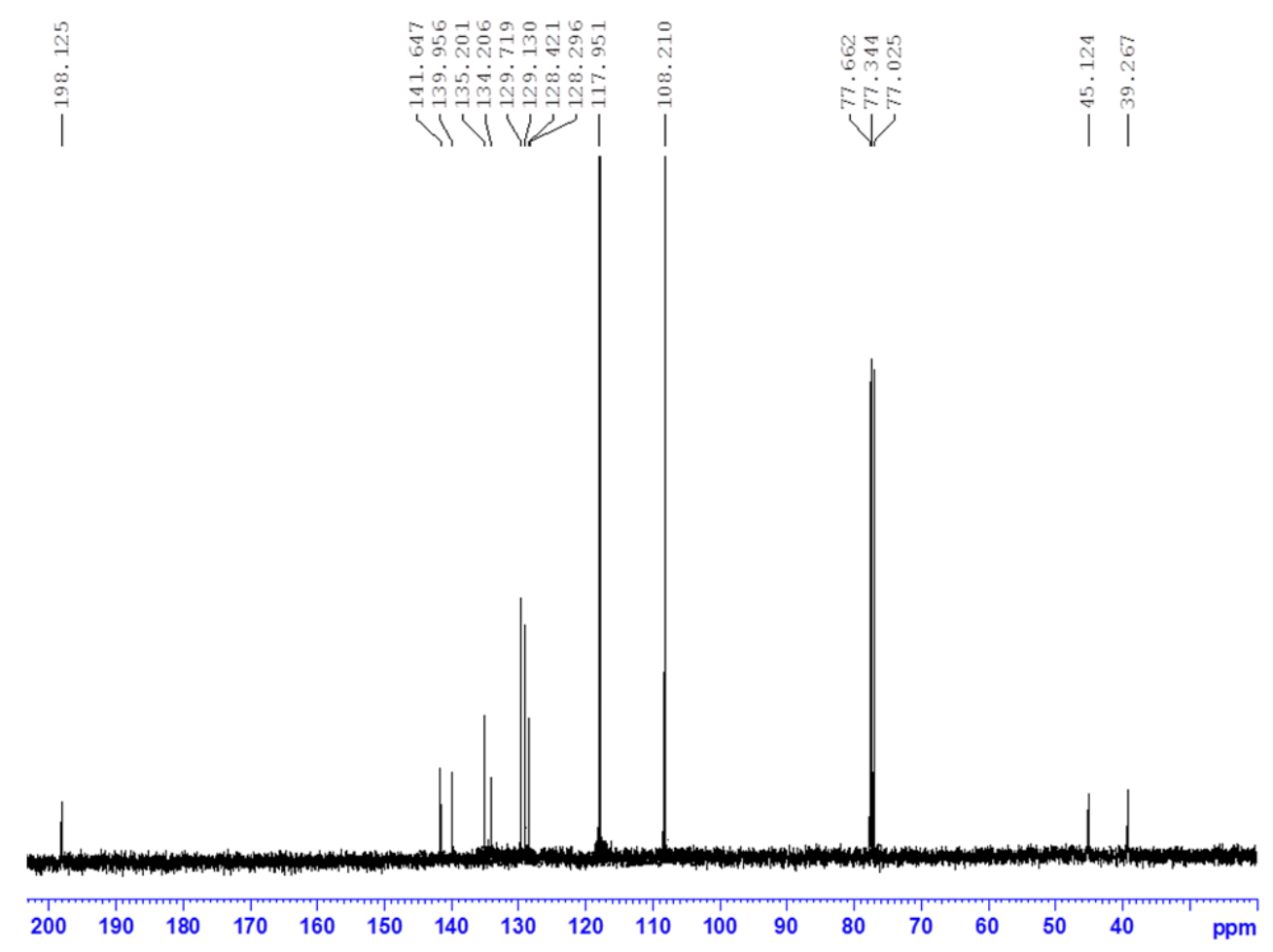

Figure S2. ${ }^{13} \mathrm{C}$ NMR spectrum (100 MHz, DMSO) of $\mathbf{6 b}$ 

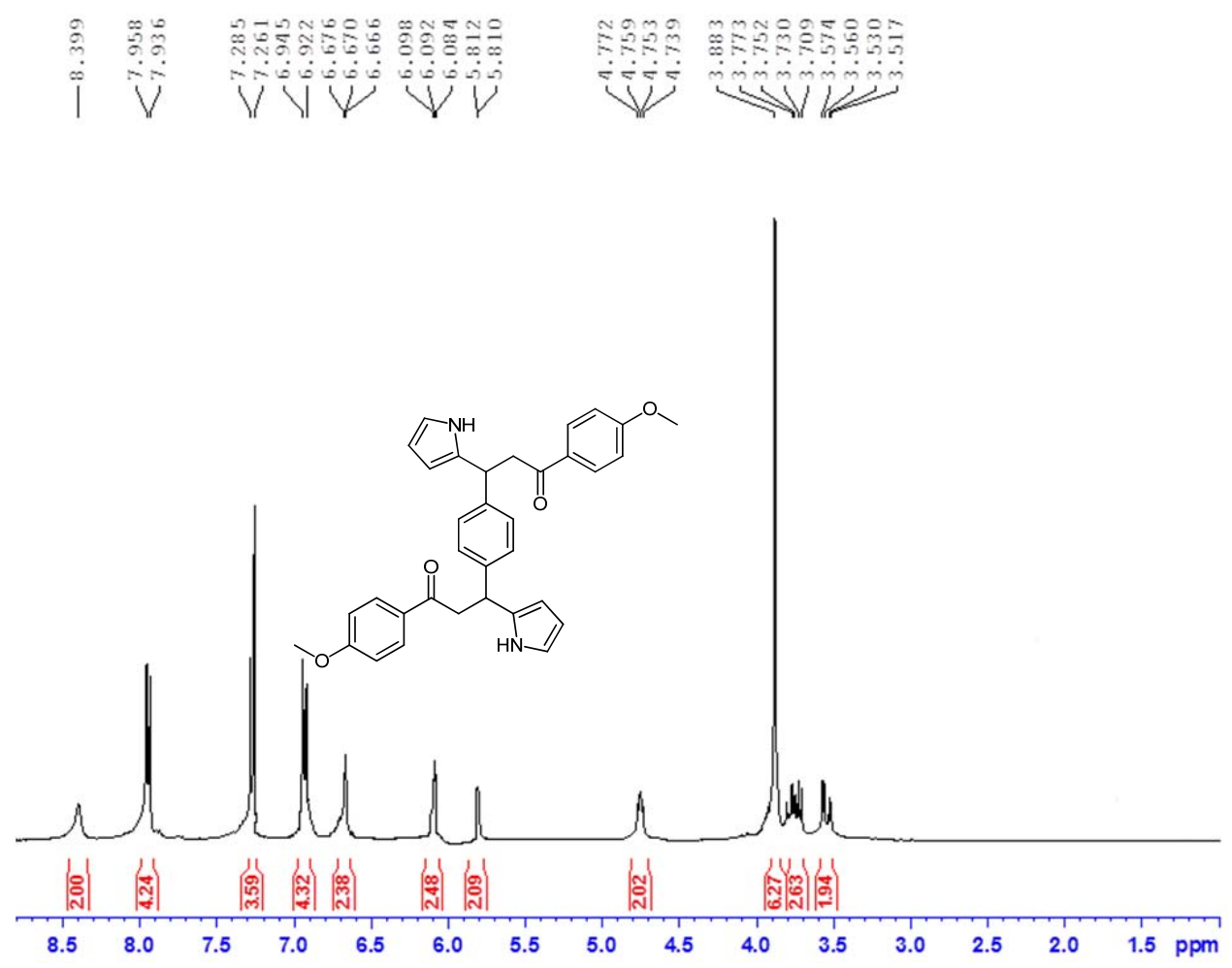

Figure S3. ${ }^{1} \mathrm{H}$ NMR spectrum (400 MHz, DMSO) of $\mathbf{6 c}$

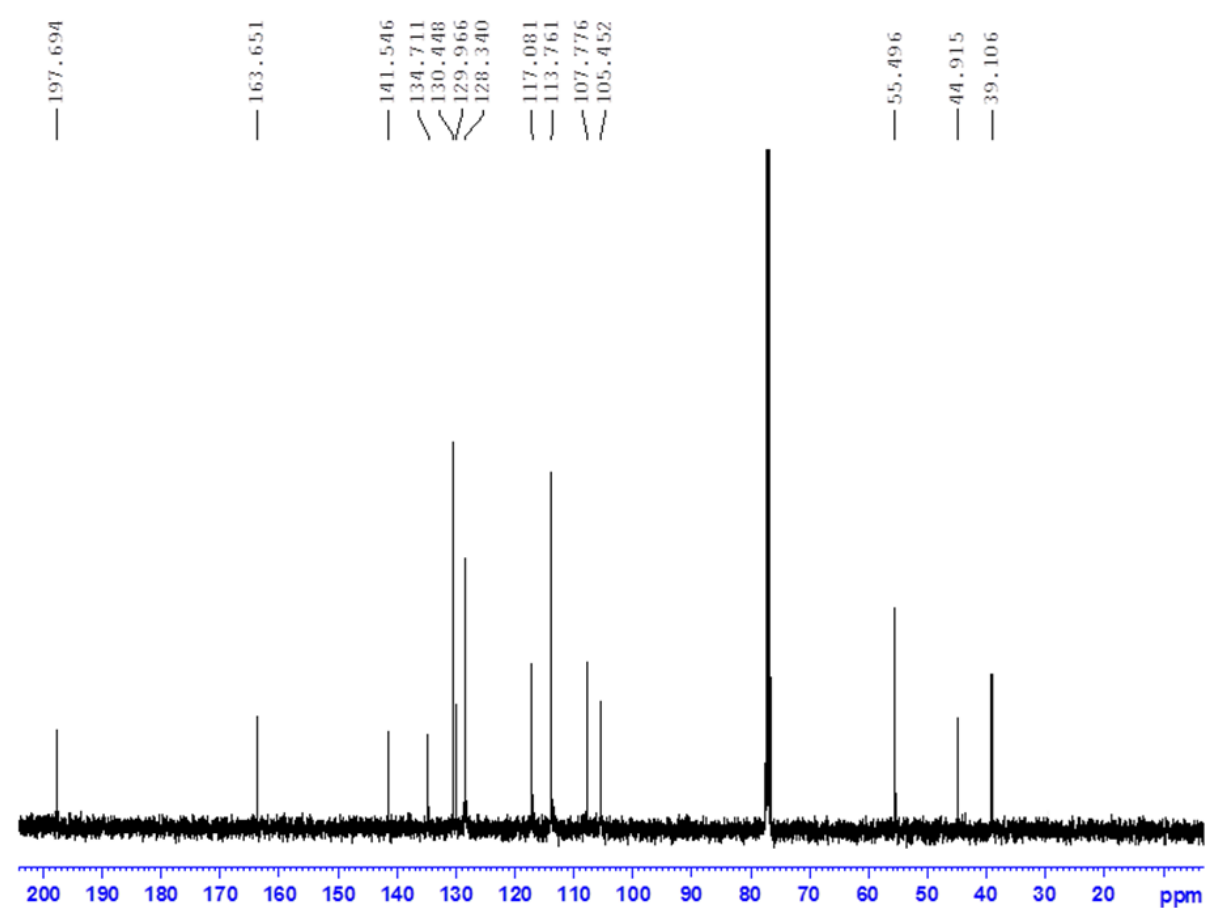

Figure S3. ${ }^{13} \mathrm{C}$ NMR spectrum (100 MHz, DMSO) of $\mathbf{6 c}$ 


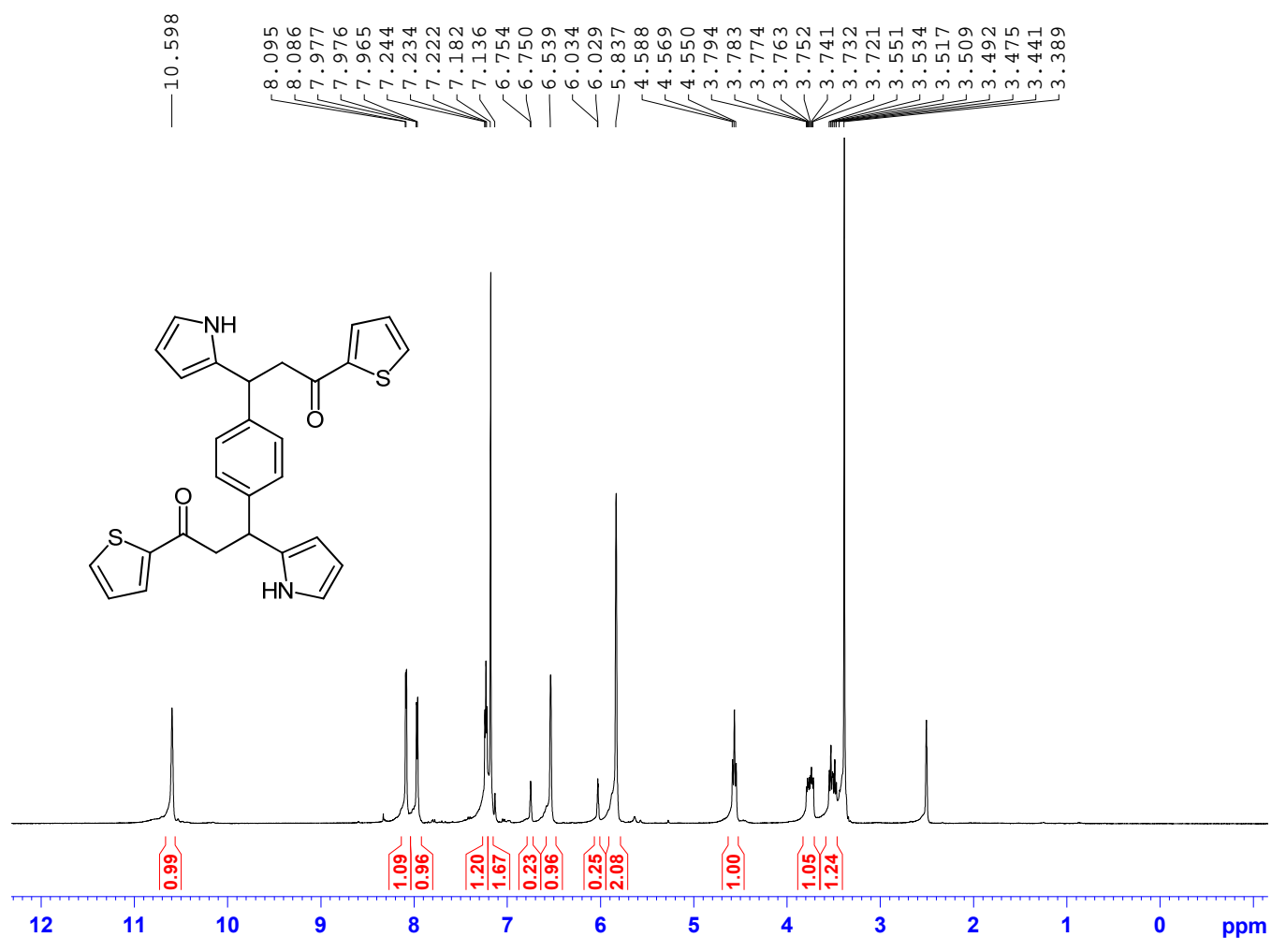

Figure S4. ${ }^{13} \mathrm{C}$ NMR spectrum (100 MHz, DMSO) of $6 \mathbf{e}$

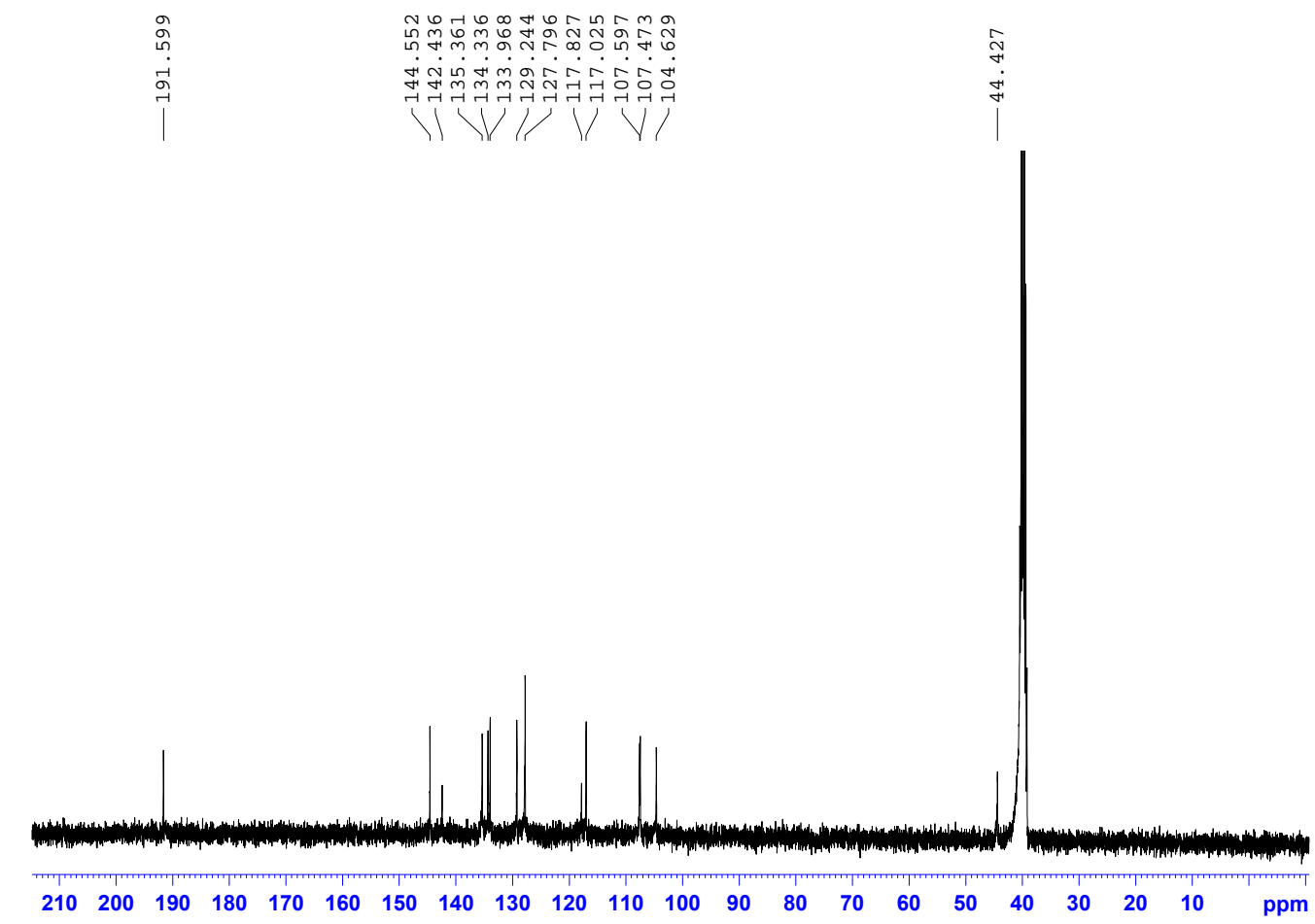

Figure S4. ${ }^{13} \mathrm{C}$ NMR spectrum (100 MHz, DMSO) of $6 \mathbf{e}$ 


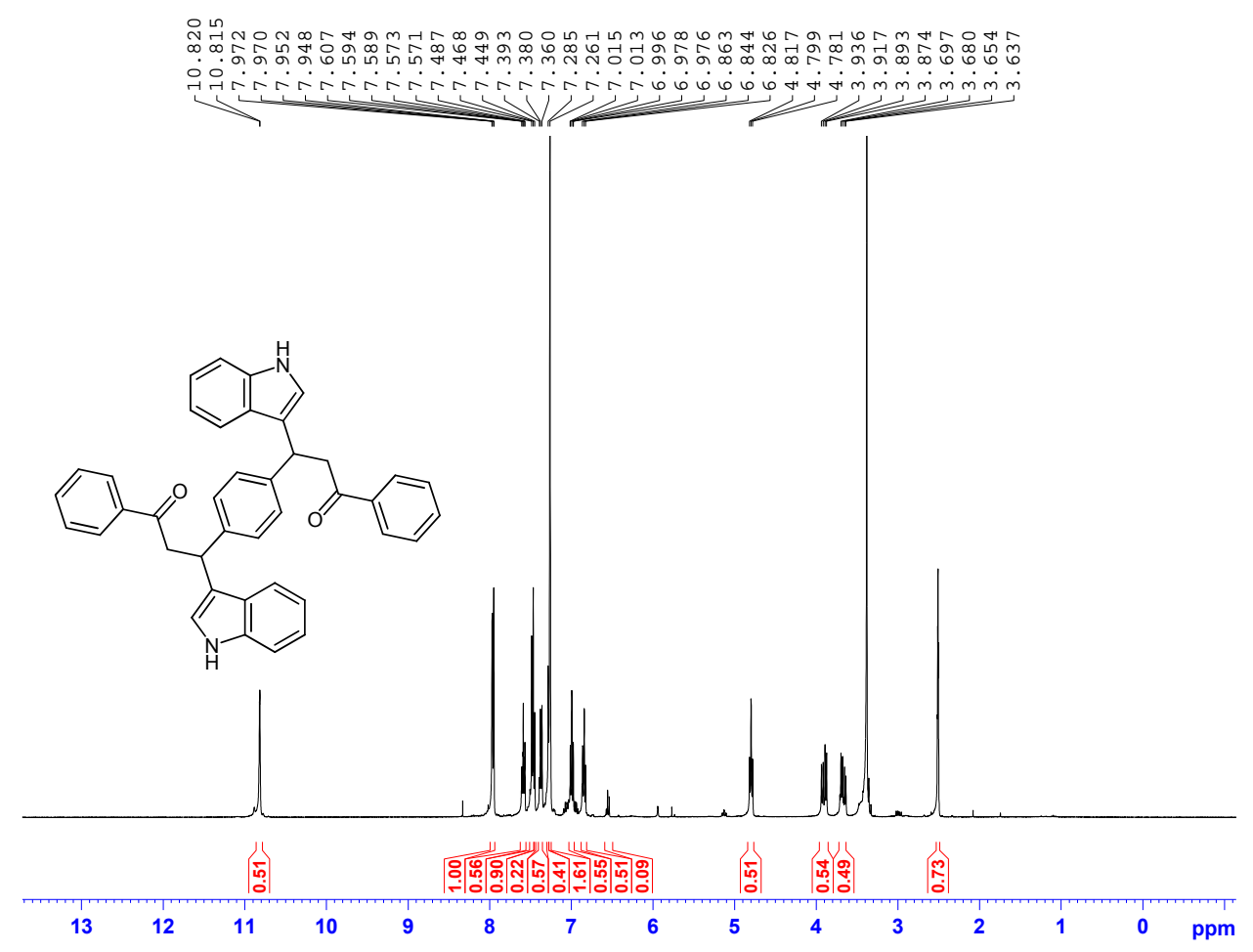

Figure S5. ${ }^{1} \mathrm{H}$ NMR spectrum (400 MHz, DMSO) of 8 a

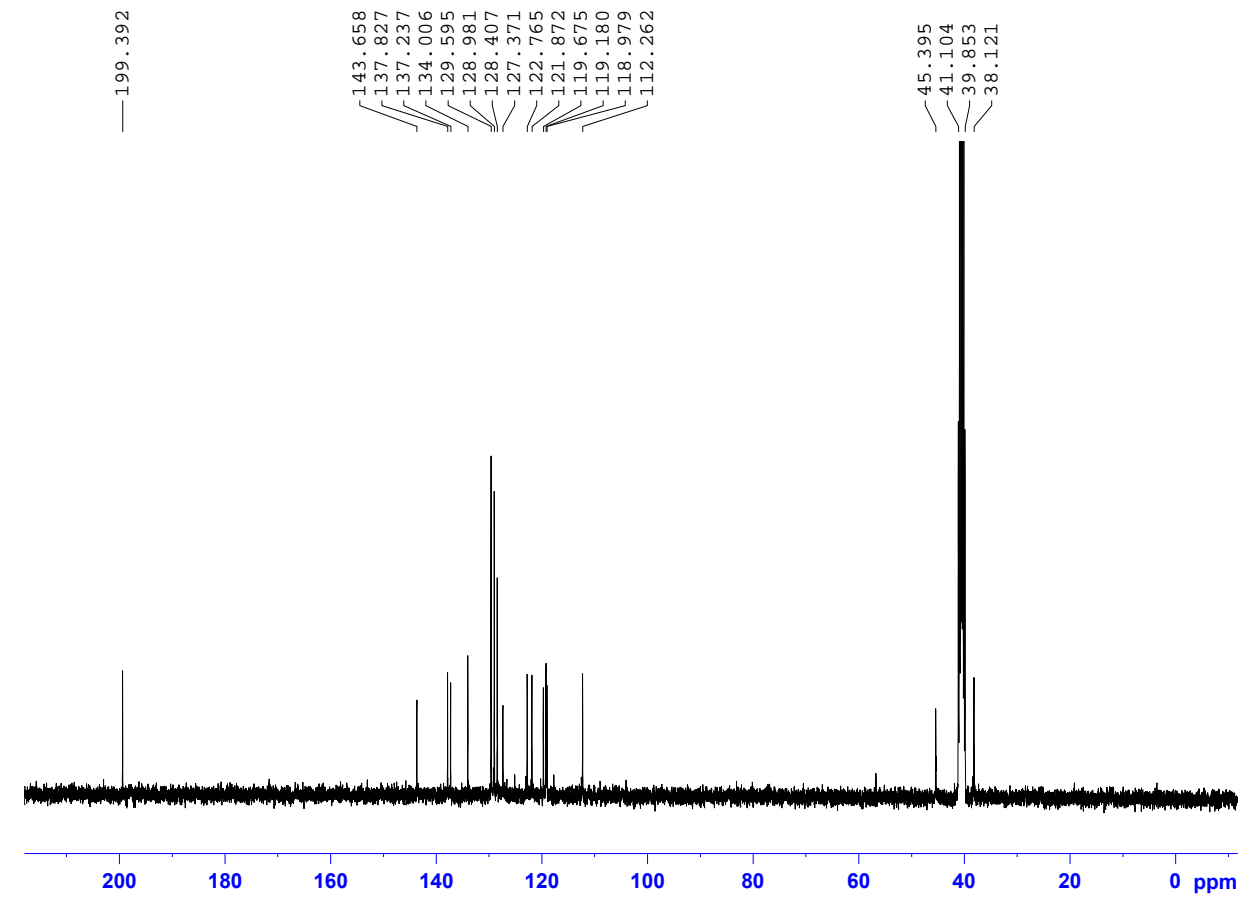

Figure S5. ${ }^{13} \mathrm{C}$ NMR spectrum $(100 \mathrm{MHz}, \mathrm{DMSO})$ of $\mathbf{8 a}$ 


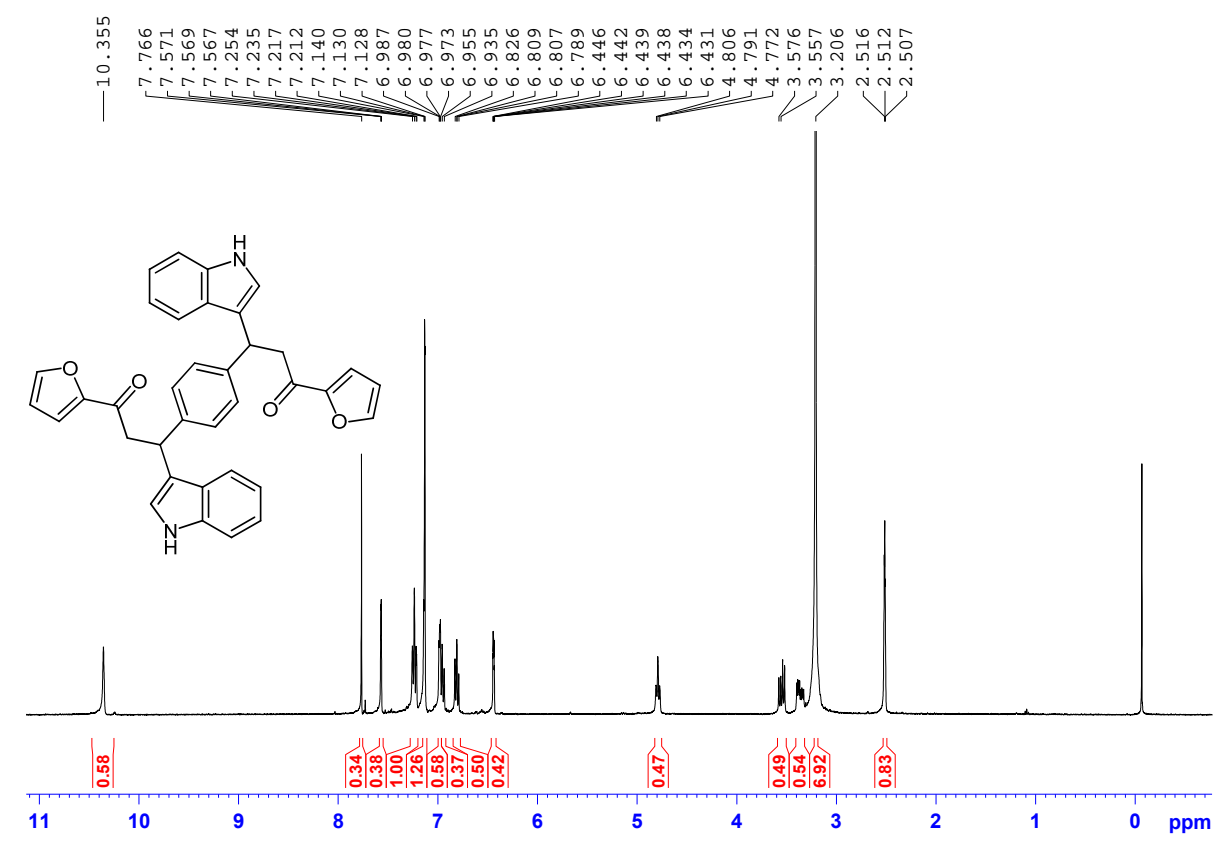

Figure S6. ${ }^{1} \mathrm{H}$ NMR spectrum (400 MHz, DMSO) of $8 \mathbf{e}$

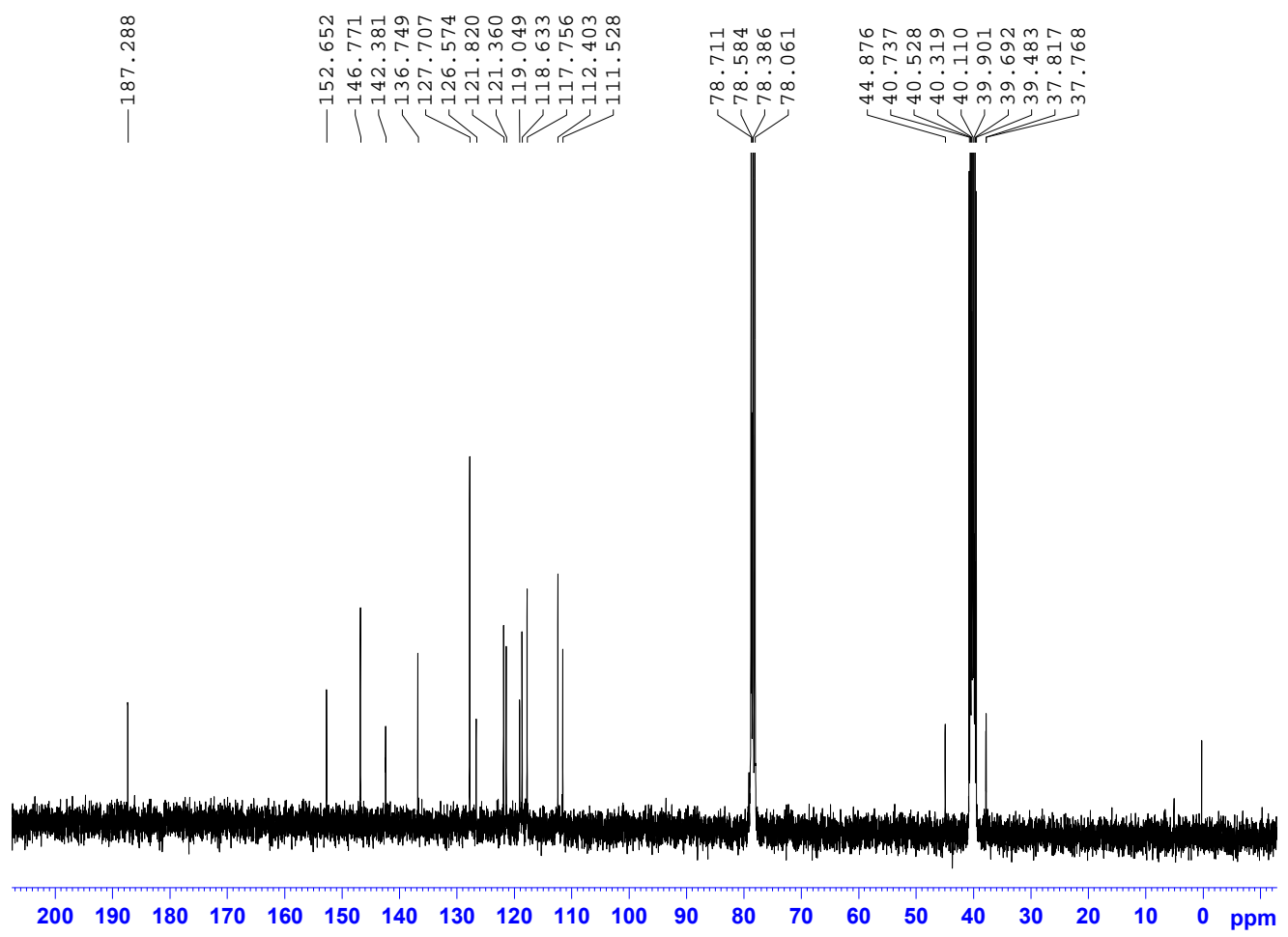

Figure S6. ${ }^{13} \mathrm{C}$ NMR spectrum (100 MHz, DMSO) of 8e 